\title{
Large Binocular Telescope observations of new six compact star-forming galaxies with [Ne v] $\lambda 3426 \AA$ emission
}

\author{
Y. I. Izotov ${ }^{1}$, T. X. Thuan ${ }^{2}$ and N. G. Guseva ${ }^{1}$ \\ ${ }^{1}$ Bogolyubov Institute for Theoretical Physics, National Academy of Sciences of Ukraine, 14-b Metrolohichna str., Kyiv, 03143, Ukraine, \\ yizotov@bitp.kiev.ua,nguseva@bitp.kiev.ua \\ ${ }^{2}$ Astronomy Department, University of Virginia, P.O. Box 400325, Charlottesville, VA 22904-4325, USA, \\ txt@virginia.edu
}

28 September 2021

\begin{abstract}
We report the discovery of [Ne v] $\lambda 3426$ emission, in addition to He II $\lambda 4686$ emission, in six compact star-forming galaxies. These observations considerably increase the sample of eight such galaxies discovered earlier by our group. For four of the new galaxies, the optical observations are supplemented by near-infrared spectra. All galaxies, but one, have $\mathrm{H}$ II regions that are dense, with electron number densities of $\sim 300-700 \mathrm{~cm}^{-3}$. They are all characterised by high $\mathrm{H} \beta$ equivalent widths $\mathrm{EW}(\mathrm{H} \beta) \sim 190-520 \AA$ and high $\mathrm{O}_{32}=[\mathrm{O}$ III $] \lambda 5007 /[\mathrm{O}$ II $] \lambda 3727$ ratios of $10-30$, indicating young starburst ages and the presence of high ionization radiation. All are low-metallicity objects with $12+\log \mathrm{O} / \mathrm{H}=7.46-7.88$. The spectra of all galaxies show a low-intensity broad component of the $\mathrm{H} \alpha$ line and five out of six objects show Wolf-Rayet features. Comparison with photoionization models shows that pure stellar ionization radiation from massive stars is not hard enough to produce such strong [Ne V] and He II emission in our galaxies. The $[\mathrm{Ne} \mathrm{v}] \lambda 3426 / \mathrm{He}$ II $\lambda 4686$ flux ratio of $\sim 1.2$ in $\mathrm{J} 1222+3602$ is consistent with some contribution of active galactic nucleus ionizing radiation. However, in the remaining five galaxies, this ratio is considerably lower, $\lesssim 0.4$. The most plausible models are likely to be non-uniform in density, where $\mathrm{He}$ II and $[\mathrm{NeV}]$ lines are emitted in low-density channels made by outflows and illuminated by harder ionizing radiation from radiative shocks propagating through these channels, whereas [O III] emission originates in denser regions exposed to softer stellar ionizing sources.
\end{abstract}

Key words: galaxies: dwarf - galaxies: starburst - galaxies: ISM - galaxies: abundances.

\section{INTRODUCTION}

The presence of hard radiation in dwarf star-forming galaxies (SFGs) has long been known thanks to the detection of a relatively strong nebular He II $\lambda 4686$ emission line in some of them. This line can serve as a probe of extremeUV ionizing radiation with photon energies higher than 4 ryd $(54 \mathrm{eV})$, corresponding to wavelengths of $\leqslant 228 \AA$ (Pakull \& Angebault 1986).

The flux of this line has been shown to increase with decreasing metallicity of the ionized gas (e.g. Garnett et al. 1991; Guseva, Izotov \& Thuan 2000; Izotov \& Thuan 2004; Thuan \& Izotov 2005). Presently, the lowest-metallicity galaxies with known He II $\lambda 4686$ emission are $\mathrm{J} 1631+4426$ with $12+\log \mathrm{O} / \mathrm{H}=6.90$ from the wide-field optical imaging data obtained with the Subaru/Hyper Suprime-Cam (HSC) (Kojima et al. 2021), and two galaxies selected in the Sloan Digital Sky Survey (SDSS) and later observed with the Large Binocular Telescope (LBT), J0811+4730 with $12+\log \mathrm{O} / \mathrm{H}=6.98$ (Izotov et al. 2018) and $\mathrm{J} 1234+3901$ with $12+\log \mathrm{O} / \mathrm{H}=7.04$ (Izotov et al. 2019).

Other high ionization emission lines of heavy elements are also seen in the spectra of some dwarf SFGs. Thus, the $[\mathrm{FeV}] \lambda 4227$ emission line was first detected by Fricke et al. (2001) and Izotov. Chaffee \& Schaerer (2001) in the blue compact dwarf (BCD) galaxies Tol 1214-277 and SBS 0335-052E, respectively. The presence of this line also requires ionizing radiation with photon energies in excess of 4 ryd. Later, this line was detected in many more SFGs (e.g. Thuan \& Izotov 2005; Izotov, Thuan \& Guseva 2017) with fluxes several times smaller than that of the He II $\lambda 4686$ emission line. As of today, the lowest-metallicity galaxy with known $[\mathrm{Fev}] \lambda 4227$ emission is the SFG J1234+3901 with $12+\log \mathrm{O} / \mathrm{H}=7.04$ (Izotov et al. 2019).

Furthermore, Izotov et al. (2001) found [Fe VI] - [Fe VII] 
emission in the BCD SBS 0335-052E, implying that it contains hard radiation with photon energies above ionization potentials of 5.5 ryd (i.e. $75 \mathrm{eV}$ ) for $\mathrm{Fe}^{5+}$, corresponding to wavelengths of radiation below $165 \AA$, and above 7.3 ryd (or $100 \mathrm{eV}$ ) for $\mathrm{Fe}^{6+}$, corresponding to wavelengths of radiation below $90 \AA$.

Another very high-ionization line is the $[\mathrm{Ne} \mathrm{v}] \lambda 3426$ emission line which, along with the He II $\lambda 4686$ emission line, is the subject of this paper. It was first discovered by Izotov et al. (2004a) in the BCD Tol 1214-277. Later, Thuan \& Izotov (2005) and Izotov, Thuan \& Privon (2012) found this emission line in seven more dwarf SFGs. This line requires the presence of extreme-UV radiation with photon energies above $7.1 \mathrm{ryd}$, (or $\geqslant 97 \mathrm{eV}$ ), corresponding to wavelengths of radiation $\lambda \leqslant 128 \AA$. It is often seen in highexcitation Seyfert 2 galaxies, where it can be considerably stronger than the He II $\lambda 4686$ emission line. This situation is contrary to the one in SFGs where $[\mathrm{NeV}]$ is invariably weaker than He II. The higher strength of [Ne V] in Seyfert 2 (Sy2) galaxies is caused partly by the high metallicity of the interstellar medium (ISM) of Sy2 galaxies and, possibly, by the harder non-thermal radiation produced by an active galactic nucleus (AGN), compared to that of SFGs.

The origin of the extreme-UV radiation in dwarf SFGs remains unclear. Several mechanisms for producing hard ionizing radiation have been proposed, such as AGNs (Izotov \& Thuan 2008), intermediate-mass black holes $\left(\gtrsim 100 \mathrm{M}_{\odot}\right)$, formed from supermassive $\left(>300 \mathrm{M}_{\odot}\right)$ stars (Kojima et al. 2021), Wolf-Rayet (WR) stars (Schaerer 1996), high-mass X-ray binaries (Garnett et al. 1991; Schaerer, Fragos \& Izotov 2019), and fast radiative shocks (Dopita \& Sutherland 1996; Izotov et al. 2004a, 2012). However, a large data base is needed to confront models with observations. In fact, $[\mathrm{NeV}] \lambda 3426$ emission has been detected only in eight SFGs (Izotov et al. 2004a; Thuan \& Izotov 2005; Izotov et al. 2012), while He II $\lambda 4686$ emission line has been detected in many more (several thousands) SFGs (Guseva et al. 2000; Thuan \& Izotov 2005; Shirazi \& Brinchmann 2012). The detection of [Ne v] $\lambda 3426$ emission line requires a large telescope equipped with spectrographs operating efficiently in the near-UV range.

The BCD SBS 0335-052E, with an oxygen abundance $12+\log \mathrm{O} / \mathrm{H}=7.30$, is the lowest-metallicity SFG with detected $[\mathrm{Ne} \mathrm{V}\rceil$ emission. Izotov et al. 2001, 2006b) and Thuan \& Izotov (2005) have discussed the origin of He II and $[\mathrm{NeV}]$ emission in that object. Those authors found that the BCD shows a HeII emission which is spatially distinct from the hydrogen $\mathrm{H} \alpha$ and $\mathrm{H} \beta$ emission, and which extends over a broader area (Izotov et al. 2006b). They thus concluded that the main source of hard radiation is not stars, but more likely radiative shocks. Furthermore, the presence of [Ne V] emission in SBS 0335-052E strengthens that conclusion. Observations of other SFGs with [NeV] emission (Izotov et al. 2004a, 2012) also support the radiative shock mechanism.

Recently Kehrig et al. (2018) have reconsidered the origin of the HeII $\lambda 4686$ emission in SBS 0335-052E. They analysed several sources of ionizing radiation and ruled out significant contributions from X-ray sources, shocks and WR stars. To study the role of stellar radiation in producing He II emission, Kehrig et al. (2018) adopted the Binary Population and Spectral Synthesis (BPASS) v2.1 stellar popula- tion synthesis models by Eldridge et al. (2017). These differ from other models, e.g. STARBURST99 (Leitherer et al. 1999, 2014), as they take into account binary stellar evolution. They found that the He II emission of SBS 0335-052E can only be produced by either single, rotating metal-free stars or a binary population with $Z \sim 10^{-5}$ and a 'top-heavy' Initial Mass Function (IMF). However, such a discrepancy between the predicted metallicity $\left(0\right.$ or $\left.10^{-5}\right)$ and the observed ISM metallicity (about $1 / 25$ solar) appears to be too large to be reasonable, even for the most metal-deficient galaxies (about $1 / 50$ solar).

In this paper, we present $\mathrm{LBT}^{1}$ spectroscopic observations of six compact SFGs. These observations are part of a long-term LBT spectroscopic program to study compact SFGs with high-excitation H II regions. Our sample consists of six SFGs with detected $[\mathrm{Ne} \mathrm{v}] \lambda 3426$ emission. Five of them, excluding HS 1851+6933, are SDSS objects. Coordinates, redshifts and other characteristics of the sample objects, obtained from the photometric and spectroscopic data, are shown in Table1 All of these SFGs are characterised by a high $\mathrm{O}_{32}=[\mathrm{O}$ III $] \lambda 5007 /[\mathrm{O}$ II $] \lambda 3727$ flux ratio, in the range $10-30$, indicating a high ionization parameter.

Some objects in Table1 have been discussed earlier in various contexts. Thus, Izotov, Thuan \& Guseva (2007) and Izotov \& Thuan (2008) selected J1222+3602 from the SDSS as a possible low-metallicity AGN candidate because of its very broad hydrogen low intensity emission. The study of its properties does imply that J1222+3602 is likely an AGN, but its SDSS spectrum did not show $[\mathrm{NeV}] \lambda 3426 \AA$ emission. Thuan \& Izotov (2005) analysed the SDSS spectrum of J0240-0828 and noted the presence of $[\mathrm{Fe} \mathrm{V}] \lambda 4227 \AA$ emission. As for $\mathrm{W} 1702+18$, it has been selected by Griffith et al. (2011) as a galaxy with an extremely red WISE $W 1-W 2$ colour, an indication of hot dust emission. Here, $W 1$ and $W 2$ are apparent magnitudes in the Vega system at $3.4 \mu \mathrm{m}$ and $4.6 \mu \mathrm{m}$, respectively. Those authors also presented optical spectroscopy and derived the chemical composition of the ISM in this galaxy. Lastly, the galaxy J1205+4551 was selected by Izotov et al. (2017) to be observed with the LBT because it has a high $\mathrm{O}_{32}$ ratio (23).

Here we use LBT observations to study the hard ionizing radiation in the objects listed in Table 1 extending the work of Izotov et al. (2017). The LBT observations and data reduction are described in Sect.2. We derive element abundances of the sample SFGs in Sect.3 How the properties of galaxies depend on the hard ionizing radiation intensity is considered in Sect.4 In Sect.5 we discuss the origin of hard ionizing radiation responsible for the $[\mathrm{Ne} \mathrm{v}] \lambda 3426$ and He II $\lambda 4686$ emission lines. We summarize our main results in Sect.6.

\footnotetext{
1 The LBT is an international collaboration among institutions in the United States, Italy and Germany. LBT Corporation partners are: The University of Arizona on behalf of the Arizona university system; Istituto Nazionale di Astrofisica, Italy; LBT Beteiligungsgesellschaft, Germany, representing the Max-Planck Society, the Astrophysical Institute Potsdam, and Heidelberg University; The Ohio State University, and The Research Corporation, on behalf of The University of Notre Dame, University of Minnesota and University of Virginia.
} 
Table 1. Observed characteristics of galaxies

\begin{tabular}{|c|c|c|c|c|c|c|c|c|c|c|c|c|}
\hline \multirow[b]{2}{*}{ Name } & \multirow[b]{2}{*}{ R.A.(J2000) } & \multirow[b]{2}{*}{ Dec.(J2000) } & \multirow[b]{2}{*}{$\mathrm{O}_{32}{ }^{\mathrm{c}}$} & \multirow[b]{2}{*}{$z^{\mathrm{d}}$} & \multicolumn{2}{|c|}{ SDSS $^{\mathrm{a}}$} & \multicolumn{2}{|c|}{$G A L E X^{\mathrm{a}}$} & \multicolumn{4}{|c|}{ WISE $^{\mathrm{b}}$} \\
\hline & & & & & $g$ & $M_{g}^{\mathrm{e}}$ & $F U V$ & $N U V$ & $W 1$ & $W 2$ & $W 3$ & $W 4$ \\
\hline J0240-0828 & $02: 40: 52.21$ & $-08: 28: 27.43$ & 11 & 0.0822 & 18.98 & -19.00 & 19.81 & 19.74 & 14.74 & 13.61 & 9.96 & 6.98 \\
\hline J0344-0106 & $03: 44: 59.28$ & $-01: 06: 16.89$ & 30 & 0.2707 & 21.52 & -19.30 & $\ldots$ & 21.32 & 17.36 & 16.48 & & \\
\hline $\mathrm{J} 1205+4551$ & 12:05:03.55 & $+45: 51: 50.94$ & 23 & 0.0654 & 19.79 & -17.62 & 21.04 & 20.58 & 15.14 & 13.55 & 9.91 & 7.80 \\
\hline $\mathrm{J} 1222+3602$ & $12: 22: 45.70$ & $+36: 02: 18.00$ & 16 & 0.3011 & 21.18 & -19.83 & 21.79 & 20.58 & 16.25 & 15.05 & 11.37 & 8.82 \\
\hline W $1702+18$ & 17:02:33.53 & $+18: 03: 06.44$ & 10 & 0.0425 & 17.27 & -19.39 & 19.14 & 19.11 & 14.29 & 12.04 & 7.60 & 4.93 \\
\hline HS $1851+6933$ & $18: 51: 17.16$ & $+69: 34: 25.92$ & 11 & 0.0253 & 17.00 & -18.44 & $\ldots$ & $\ldots$ & $\ldots$ & $\ldots$ & $\ldots$ & $\ldots$ \\
\hline
\end{tabular}

${ }^{a} \mathrm{AB}$ magnitudes.

bega magnitudes.

${ }^{\mathrm{c}} \mathrm{O}_{32}$ is the extinction-corrected [O $\left.\mathrm{III}\right] \lambda 5007 /[\mathrm{O}$ II] $\lambda 3727$ flux ratio in the LBT spectrum.

$\mathrm{d}_{z}$ is the redshift derived from the LBT spectrum of HS 1851+6933 and from SDSS spectra for the remaining galaxies.

eAbsolute SDSS $g$-band magnitude corrected for Milky Way extinction and adopting the distance for a flat Universe with $H_{0}=69.6 \mathrm{~km} \mathrm{~s}^{-1}, \Omega_{\mathrm{m}}=0.286$ and $\Omega_{\Lambda}=0.714$ (NED, Wright 2006).

Table 2. Journal of observations

\begin{tabular}{llccrcc}
\hline Name & \multicolumn{1}{c}{ Inst. } & Date & $\begin{array}{c}\text { Exp. } \\
(\mathrm{s})\end{array}$ & $\begin{array}{c}\text { Slit } \\
(\operatorname{arcsec})\end{array}$ & $\begin{array}{c}\text { Seeing } \\
(\operatorname{arcsec})\end{array}$ & Airmass \\
\hline J0240-0828 & MODS1 & $2013-12-07$ & 3200 & $60 \times 1.2$ & 1.8 & 1.33 \\
& LUCI1+LUCI2 & $2019-01-04$ & $1440+1440$ & $240 \times 1.0$ & 1.0 & 1.34 \\
J0344-0106 & MODS1 & $2019-10-25$ & 3600 & $60 \times 1.2$ & 1.5 & 1.27 \\
J1205+4551 & MODS1 & $2016-04-10$ & 3200 & $60 \times 1.2$ & 1.1 & 1.24 \\
& LUCI1+LUCI2 & $2021-01-09$ & $2400+2400$ & $240 \times 1.0$ & 1.0 & 1.03 \\
J1222+3602 & MODS1 & $2015-05-18$ & 3200 & $60 \times 1.2$ & 0.8 & 1.01 \\
W 1702+18 & MODS1 & $2013-06-09$ & 1800 & $60 \times 1.0$ & 1.0 & 1.08 \\
& LUCI2 & $2016-06-16$ & 1440 & $240 \times 1.0$ & 0.8 & 1.03 \\
HS 1851+6933 & MODS1 & $2013-06-07$ & 2400 & $60 \times 1.0$ & 1.0 & 1.20 \\
& LUCI2 & $2016-06-16$ & 1440 & $240 \times 1.0$ & 1.0 & 1.25 \\
\hline
\end{tabular}

\section{LBT OBSERVATIONS AND DATA REDUCTION}

\subsection{MODS observations}

We have obtained LBT long-slit spectrophotometric observations in the optical range of the six sample galaxies during the period 2013 - 2019, using the MODS1 (Multi-Object Double Spectrograph) instrument $2^{2}$ in the monocular mode.

MODS1 spectra were obtained with exposures ranging from $1800 \mathrm{~s}$ to $3600 \mathrm{~s}$, in the wavelength range $3200-$ $10000 \AA$ with 1.0 and 1.2 arcsec wide slits (Table2). The seeing changed from 0.8 to 1.8 arcsec during different nights. The airmass varied from 1.01 to 1.33 , meaning that the effect of atmospheric refraction is small for all objects (Filippenko 1982). MODS1 spectra of several spectrophotometric standard stars were obtained during the same nights with a 5 arcsec wide slit for flux calibration and correction for telluric absorption lines in the red part. Calibration frames of biases, flats and argon comparison lamps were obtained during the daytime, before or after the observations.

Bias subtraction, flat field correction, wavelength and flux calibration have been done with the MODS Basic CCD

2 This paper used data obtained with the MODS spectrographs built with funding from NSF grant AST-9987045 and the NSF Telescope System Instrumentation Program (TSIP), with additional funds from the Ohio Board of Regents and the Ohio State University Office of Research.
Reduction package MODSCCDREL $3^{3}$ and IRAF Selected segments of one-dimensional spectra of the galaxies listed in Table1 extracted in a 1.2 arcsec aperture along the spatial axis, are shown in Fig. A1 Strong emission lines can be seen in these spectra, suggesting active star formation. Some of these lines are labelled in the MODS spectrum of HS 1851+6933 (Fig.A1 - A1b). In particular, a strong [O III] $\lambda 4363$ emission line is detected with a high signal-tonoise ratio in all galaxies, allowing a reliable abundance determination of their ISM. Other striking features are the $[\mathrm{FeV}] \lambda 4227$ emission line, detected in MODS1 spectra of nearly all galaxies, with the exception of J1222+3602, and the He II $\lambda 4686$ emission line seen in all galaxies (Fig.1 Table A1 . Finally, the $[\mathrm{NeV}] \lambda 3426$ emission line is also detected in the totality of galaxies. We also note the strong broad component of the $\mathrm{H} \alpha$ emission line in the spectrum of J1222+3602 and the considerably weaker broad $\mathrm{H} \beta$ component, indicating considerable collisional excitation of the former line (Fig. A1h). As mentioned before, this galaxy has been studied by Izotov \& Thuan (2008) who concluded that it is likely a low-metallicity AGN.

\footnotetext{
3 http://www.astronomy.ohio-state.edu/MODS/Manuals/ MODSCCDRed.pdf

4 IRAF is distributed by the National Optical Astronomy Observatories, which are operated by the Association of Universities for Research in Astronomy, Inc., under cooperative agreement with the National Science Foundation.
} 


\subsection{LUCI observations}

In addition, near-infrared spectra in the $9000-13000 \AA$ wavelength range during the 2013 - 2019 period have been obtained with the LUCI1 and LUCI2 (LBT Utility Camera in the Infrared) spectrograph 5 of four out of the six sample SFGs.

These observations were aimed at detecting the He I $\lambda 10831 \AA$ emission line, the flux of which (relative to the $\mathrm{H} \beta$ flux) is very sensitive to the electron number density in the HII region. The two galaxies with the highest redshifts in our sample, J0344-0106 and J1222+3602, were not observed because the He I $\lambda 10831 \AA$ in these galaxies falls in the region of strong telluric absorption. For subtraction of the sky background, we obtained a series of equal numbers of subexposures at two positions $\mathrm{A}$ and $\mathrm{B}$ along the slit. The duration of each subexposure was $240 \mathrm{~s}$. Background subtraction has been done with the following three steps. First, frames at positions A and B were separately co-added. Then the co-added frame at position B was subtracted from the co-added A frame, resulting in a background-subtracted frame with a positive spectrum at position $\mathrm{A}$ and a negative spectrum at position B. Finally, the negative spectrum is shifted to the position of the positive spectrum and subtracted from it, producing a final spectrum. Each observation of a galaxy was supplemented by the observation of an A0 star with known brightness at two positions A and B along the slit and at a similar airmass for flux calibration and correction of the spectrum for telluric absorption. Furthermore, a sequence of frames with the Ar comparison lamp and dark current with equal exposures were obtained during the daytime. Then the co-added frame with dark current was subtracted from the co-added frames with the spectra of the Ar comparison lamp.

We note that, in the near-infrared range, very strong He I $\lambda 10831 \AA$ emission is observed in all our objects (compared to the nearby hydrogen $\mathrm{P} \gamma \lambda 10941 \AA$ emission line) (Fig. A1) indicating a high electron number density. This line and some other strong emission lines are labelled in the LUCI spectrum of HS 1851+6933 (Fig.A1p).

\subsection{Extinction-corrected emission-line fluxes}

The observed emission-line fluxes and their errors were measured using the IRAF SPLOT routine. The fluxes were measured in the flux-calibrated spectra, whereas their errors were measured in the non-flux-calibrated spectra using Poisson statistics. The errors were scaled to flux units by using the ratio of the flux to the line count in the non-flux-calibrated spectrum. Both the fluxes and errors were corrected for extinction, derived from the observed decrement of the hydrogen Balmer emission lines after their correction for underlying stellar absorption, following Izotov, Thuan \& Lipovetsky (1994). The equivalent widths of the underlying stellar Balmer absorption lines are assumed to be the same for each line. They were derived iter-

${ }^{5}$ LUCI1 and LUCI2 are mounted at the front Bent Gregorian $\mathrm{f} / 15$ focal stations of the LBT, and were designed and built by the LUCI consortium, which consists of Landessternwarte Heidelberg, MPE, MPIA, and Astronomisches Institut der Universität Bochum. atively and simultaneously with the extinction coefficient so as to reproduce the observed hydrogen Balmer decrements. The extinction-corrected fluxes are shown in Table A1 Visible and near-infrared observations have been obtained during different epochs, with different weather conditions and slit widths. Therefore, in order to link the nearinfrared observations to the optical ones, the NIR fluxes have been scaled to obtain the theoretical recombination $I(\mathrm{P} \gamma \lambda 10941) / I(\mathrm{H} \beta)$ flux ratio, after correction for extinction, and adopting the electron temperature $T_{\mathrm{e}}(\mathrm{O}$ III). The Table also includes, for each galaxy, the extinction coefficient $C(\mathrm{H} \beta)$, the rest-frame equivalent width $\mathrm{EW}(\mathrm{H} \beta)$, the equivalent width of the Balmer absorption lines, and the observed $\mathrm{H} \beta$ flux $F(\mathrm{H} \beta)$.

\section{ELEMENT ABUNDANCES}

Following Izotov et al. (2006a), we use the direct $T_{\mathrm{e}}$-method to derive the electron temperatures and element abundances. The temperature $T_{\mathrm{e}}(\mathrm{O}$ III $)$ is obtained from the [O III $] \lambda 4363 /(\lambda 4959+\lambda 5007)$ emission-line flux ratios. It is used to derive the abundances of $\mathrm{O}^{3+}, \mathrm{O}^{2+}$ and $\mathrm{Ne}^{2+}$. The abundances of $\mathrm{N}^{+}, \mathrm{O}^{+}, \mathrm{S}^{+}$and $\mathrm{Fe}^{2+}$ are derived with the electron temperature $T_{\mathrm{e}}(\mathrm{O}$ II), obtained from the relation between $T_{\mathrm{e}}(\mathrm{O}$ II $)$ and $T_{\mathrm{e}}$ (O III) (Izotov et al. 2006a), whereas the abundances of $\mathrm{S}^{2+}, \mathrm{Cl}^{2+}$ and $\mathrm{Ar}^{2+}$ are obtained with the electron temperature $T_{\mathrm{e}}$ (S III) (Izotov et al. 2006a). The electron number densities $N_{\mathrm{e}}(\mathrm{S}$ II) are calculated from the [S II] $\lambda 6717 / \lambda 6731$ emission line flux ratios.

The electron number density can also be derived from the fluxes of He I emission lines (Izotov, Thuan \& Guseva 2014). In general, emission in He I lines comes from recombination processes. However, it is modified by two nonrecombination mechanisms, collisional and fluorescent excitation. The relative role of these two processes is different depending on the He I emission line. In particular, the He I $\lambda 6678$ emission line flux is least sensitive to both the collisional and fluorescent excitations, whereas the flux of He I $\lambda 10831$ emission line is not sensitive to fluorescent excitation, but is extremely sensitive to the electron number density. Thus, the HeI $\lambda 10831 / \lambda 6678$ flux ratio is a good measure of the electron number density in a $\mathrm{He}^{+}$zone. To derive $N_{\mathrm{e}}(\mathrm{He}$ I), we adopt the Izotov, Stasińska \& Guseva (2013) approximations of the Porter et al. (2013) He I emissivities.

The total oxygen abundance $\mathrm{O} / \mathrm{H}$ is derived as the sum of the $\mathrm{O}^{+} / \mathrm{H}^{+}, \mathrm{O}^{2+} / \mathrm{H}^{+}$and $\mathrm{O}^{3+} / \mathrm{H}^{+}$ionic abundances using the relations of Izotov et al. (2006a). For the ionic and total abundances of nitrogen, neon, sulfur, chlorine, argon and iron, we also use the relations of Izotov et al. (2006a).

The electron temperatures, electron number densities, ionic abundances, ionization correction factors, ionic and total $\mathrm{N}, \mathrm{O}, \mathrm{Ne}, \mathrm{S}, \mathrm{Cl}, \mathrm{Ar}$ and $\mathrm{Fe}$ abundances are presented in TableA2 All observed galaxies have high $T_{\mathrm{e}}(\mathrm{O}$ III $)$, in the range $16000-20600 \mathrm{~K}$, and atypically high $N_{\mathrm{e}}(\mathrm{S}$ II $)$, in the range $\sim 300-700 \mathrm{~cm}^{-3}$ for five galaxies. Only in one galaxy, J0344-0106, $N_{\mathrm{e}}(\mathrm{S}$ II $)$ is as low as $28 \mathrm{~cm}^{-3}$. However, this galaxy is the faintest in the sample, with an apparent SDSS $g$-band magnitude of $21.52 \mathrm{mag}$ (Table1). Therefore, its spectrum is noisier compared to other galaxies, and the determination of its $N_{\mathrm{e}}(\mathrm{S}$ II) is somewhat uncertain. The elec- 


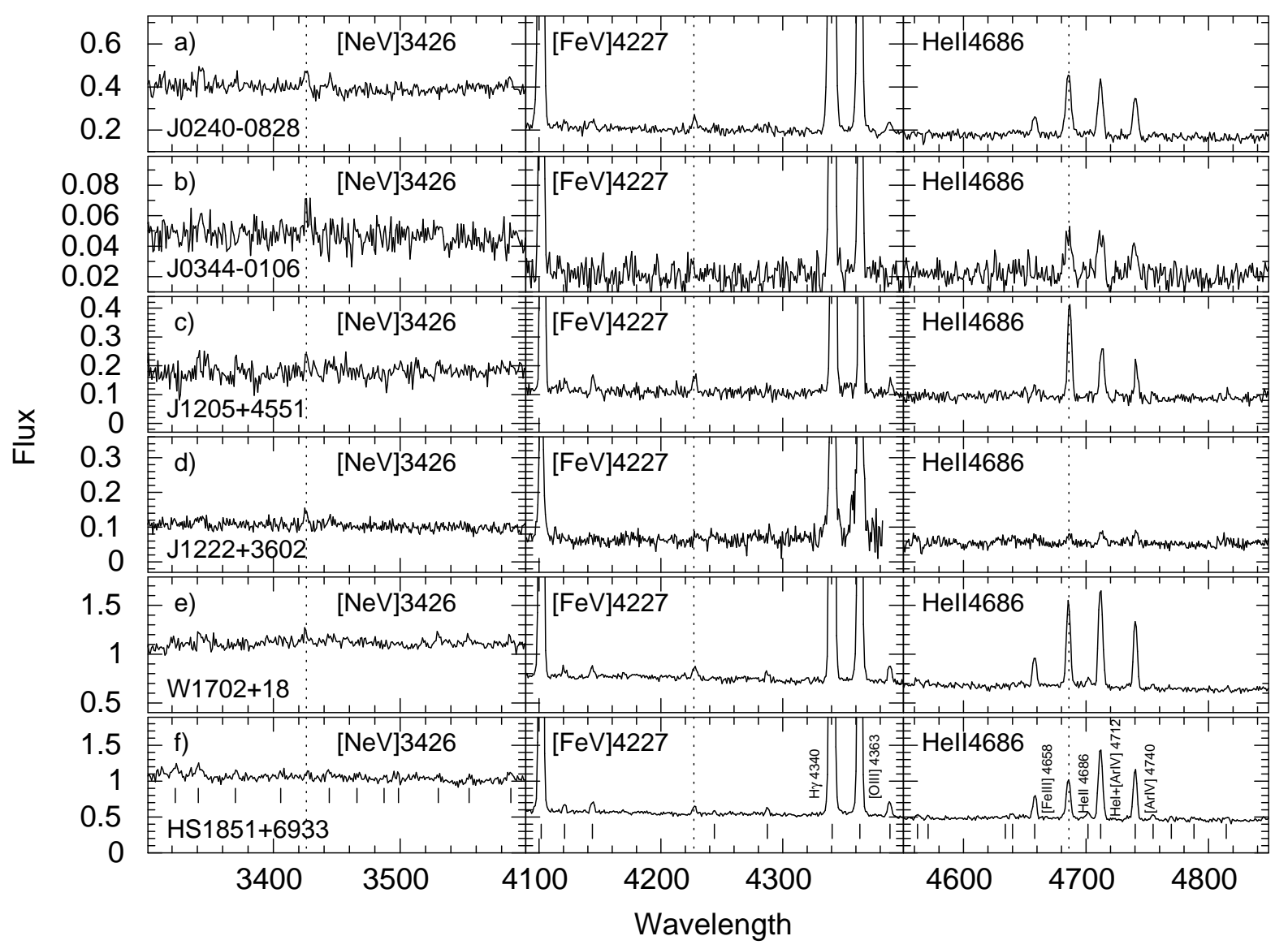

Figure 1. Segments of non-corrected for extinction restframe LBT spectra with the $[\mathrm{Nev}] \lambda 3426$ emission line (left panels), the [Fev] $\lambda 4227$ emission line (middle panels) and the He II $\lambda 4686$ emission line (right panels). Their locations are indicated by vertical dotted lines. Locations of other weak and strong emission lines in the UV range of the HS 1851+6933 spectrum (Table A1) are indicated in f) by short vertical lines. The brightest lines are labelled in $\mathbf{f}$ ).

tron number densities $N_{\mathrm{e}}(\mathrm{He}$ I) derived for the four galaxies with available near-infrared observations are also high, in agreement with those obtained from the [S II] emission-line flux ratios.

How do our abundance measurements compare with previous determinations? We derive a nebular oxygen abundance $12+\log \mathrm{O} / \mathrm{H}$ in $\mathrm{W} 1702+18$ of $7.75 \pm 0.02$, somewhat higher than the value of $7.63 \pm 0.06$ obtained by Griffith et al. (2011). The oxygen abundance in J1222+3602 of $7.69 \pm 0.02$ is somewhat lower than the value of $7.88 \pm 0.05$ obtained by Izotov \& Thuan (2008). Our determination of $12+\log \mathrm{O} / \mathrm{H}$ $=7.88 \pm 0.02$ for $\mathrm{J} 0240-0828$ is in good agreement with the value of $7.89 \pm 0.02$ obtained by Thuan \& Izotov (2005).

The Ne/O, S/O, Ar/O and Fe/O abundance ratios for these galaxies (Table A2 Fig. 2) follow the relations obtained for other low-metallicity SFGs (grey dots, black filled circles, encircled black filled circles, open circles, asterisks), and the two most metal-deficient SFGs known with $12+\log \mathrm{O} / \mathrm{H}<7.0$, J1631+4426 (cyan symbol, Kojima et al. 2021) and J0811+4730 (magenta symbol, Izotov et al. 2018), which are shown for comparison. Some of the comparison galaxies, which are shown by asterisks, open circles and encircled symbols (excluding the cyan encircled circle), have been observed in the wavelength range covering $[\mathrm{Nev}] \lambda 3426$ emission line, but with no detec- tion. We note that, although $[\mathrm{Ne} \mathrm{v}]$ emission is not detected in $\mathrm{J} 0811+4730$ and has not been observed in $\mathrm{J} 1631+4426$, a relatively strong He II $\lambda 4686$ emission line, with an intensity of $\sim 2$ per cent that of the $\mathrm{H} \beta$ emission line, is seen in both galaxies. Fig. 2 shows that J0811+4730 (magenta symbol) follows well sequences for other SFGs, whereas J1631+4426 (cyan symbol) deviates somewhat more from these sequences.

On the other hand, the $\mathrm{Cl} / \mathrm{O}$ abundance ratios for galaxies with $[\mathrm{Ne} \mathrm{V}]$ emission are somewhat lower than those of other SFGs. We also note that the N/O abundance ratios for the new galaxies with detected $[\mathrm{NeV}]$ emission discussed in this paper are systematically higher (by $0.4-0.9 \mathrm{dex}$ ) than the average values for the low-metallicity SFGs from the HeBCD sample used for the determination of primordial He abundance (Izotov \& Thuan 2004; Izotov et al. 2004b), in the same range of oxygen abundances (black filled circles). However, previous galaxies with detected [Nev] emission from Izotov et al. (2004a), Thuan \& Izotov (2005) and Izotov et al. (2012) have, with the exception of one galaxy, $\mathrm{N} / \mathrm{O}$ ratios that are similar to those of the HeBCD sample.

The rapid decrease of the $\mathrm{Fe} / \mathrm{O}$ abundance ratio with increasing oxygen abundance in Fig. 2f is remarkable. Izotov et al. (2006a) interpret this behaviour as a depletion of iron, and in a lesser extent of oxygen due to the formation 


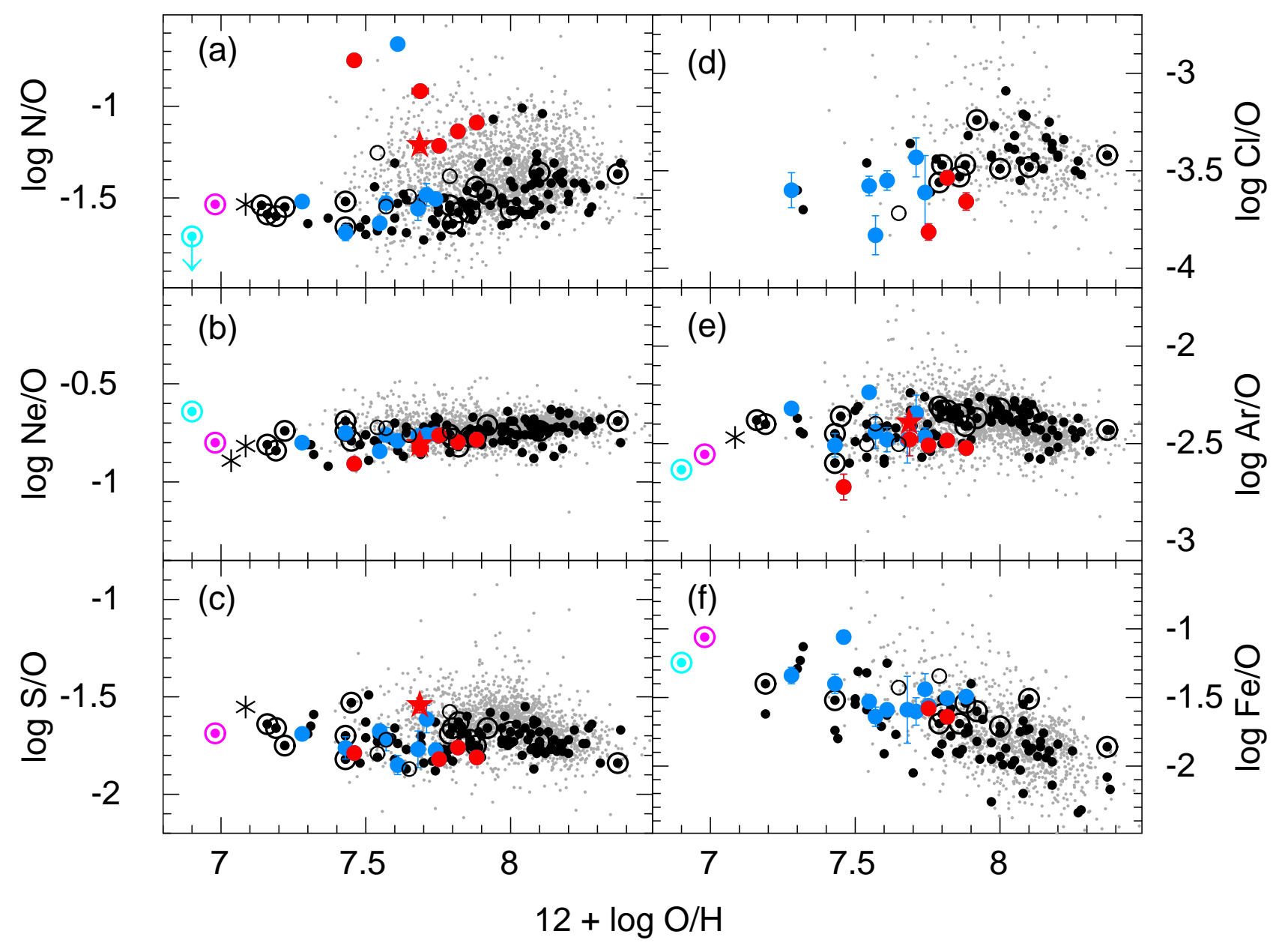

Figure 2. Dependences of different elemental abundance ratios on the oxygen abundance $12+\log \mathrm{O} / \mathrm{H}$. The new galaxies in this paper with [Nev] emission, excluding J1222+3602, are shown by red filled circles. J1222+3602, a dwarf AGN, is shown by a red filled star. Previously known galaxies with [Ne v] emission (Izotov et al. 2004a; Thuan \& Izotov 2005; Izotov et al. 2012) are shown by blue filled circles. For all these SFGs (blue and red symbols), we show $1 \sigma$ error bars. However, in most cases, the error bars are so small compared to the size of the circles that they are not easily seen. For comparison, we show two most metal-deficient SFGs, J1631+4426 (cyan symbol, Koiima et al. 2021) and J0811+4730 (magenta symbol, Izotov et al. 2018), with $12+\log \mathrm{O} / \mathrm{H}<7.0$, two other most metal-deficient SFGs, J1234+3901 and J2229+2725, with $12+\log \mathrm{O} / \mathrm{H}$ in the range $7.0-7.1$ (asterisks, Izotov et al. 2019, 2021), SFGs from the SDSS DR16 with $[\mathrm{O} \mathrm{II}] \lambda 4363$ fluxes measured with an accuracy better than $4 \sigma$ (grey dots), and the HeBCD sample from Izotov \& Thuan (2004) and Izotov et al. (2004b), used for primordial helium abundance determination (black filled circles). Encircled black filled circles are those galaxies from the HeBCD sample, which have been observed in the wavelength range covering [Ne v] $\lambda 3426$ emission line, but with no detection of this line (Thuan \& Izotov 2005). Four compact SFGs, observed with the LBT, with extreme $\mathrm{O}_{32}=23-43$ and non-detected [Ne v] $\lambda 3426$ emission line are shown by open circles (Izotov. Thuan \& Guseva 2017).

of dust grains, which is more effective at higher metallicities. This is supported by the slight increase of the Ne/O abundance ratio with increasing oxygen abundance due to depletion of oxygen, whereas the noble element neon is not depleted. Contrary to that point of view, Kojima et al. (2021) have analysed the data for the extremely metal-deficient galaxies $\mathrm{J} 1631+4426$ and $\mathrm{J} 0811+4730$, and suggested that their very high $\mathrm{Fe} / \mathrm{O}$ ratios are due to the increased Fe production by very massive stars, with masses $>300 \mathrm{M}_{\odot}$, which may have existed in these galaxies. However, that suggestion may not work because similarly high $\mathrm{Fe} / \mathrm{O}$ are found in some compact SFGs with much higher metallicities (Fig.2f).

In conclusion, the overall abundance patterns of the galaxies with detected $[\mathrm{NeV}]$ emission show no anomalies compared to the bulk of compact SFGs. An exception is for the $\mathrm{N} / \mathrm{O}$ ratio. The $\mathrm{N} / \mathrm{O}$ ratio in about half of the galaxies with a detected $[\mathrm{NeV}] \lambda 3426$ emission line is similar to that in galaxies without [ $\mathrm{NeV}]$ emission. On the other hand, the $\mathrm{N} / \mathrm{O}$ abundance ratio in the remaining half is by a factor of $\sim 3-10$ higher (Fig. 2a). Izotov et al. (2006b) assumed that the N/O enhancement of the interstellar medium can be due to stellar winds from Wolf-Rayet stars enriched in nitrogen. They argued that chemical evolution models of the well-mixed uniform medium cannot reproduce high $\mathrm{N} / \mathrm{O}$ ratios. On the other hand, the considerable N/O enhancement can be achieved adopting a medium with dense nitrogenenriched clumps around Wolf-Rayet stars embedded in the low-density interstellar medium. 

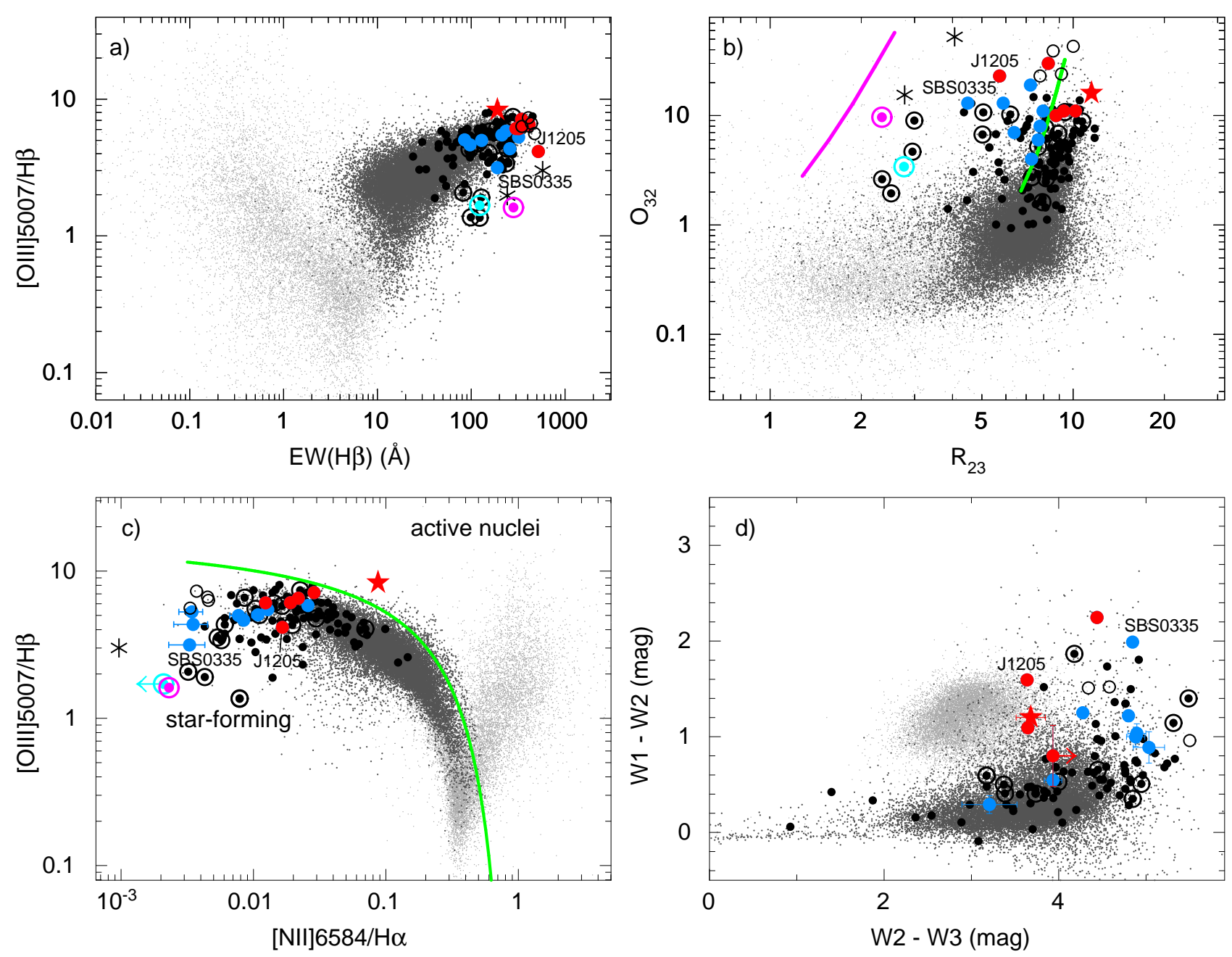

Figure 3. a) $[\mathrm{O} \mathrm{III}] \lambda 5007 / \mathrm{H} \beta$ flux ratio vs equivalent width $\mathrm{EW}(\mathrm{H} \beta)$. The new galaxies in this paper with [Ne v] emission, excluding $\mathrm{J} 1222+3602$, are shown by red filled circles. J1222+3602 is shown by a red filled star. Previously known galaxies with [Ne v] emission (Izotov et al. 2004a; Thuan \& Izotov 2005; Izotov et al. 2012) are shown by blue filled circles. For comparison, a sample of SDSS SFGs is represented by dark-grey dots, SDSS DR7 galaxies including AGN are shown by light-grey dots. Meaning of other symbols is the same as in Fig.2 b) $\mathrm{O}_{32}=[\mathrm{O}$ III $] \lambda 5007 /[\mathrm{O}$ II $] \lambda 3727$ flux ratio vs. $\mathrm{R}_{23}=([\mathrm{O}$ II $] \lambda 3727+[\mathrm{O} \mathrm{III}] \lambda 4959+[\mathrm{O}$ III $] \lambda 5007) / \mathrm{H} \beta$ flux ratio. The dark-grey and light-grey dots are the same as in a). Loci of values modelled with CLOUDY code for various ionization parameters in the range of $\log U$ between -1.3 and -3.0 and adopting constant $12+\log \mathrm{O} / \mathrm{H}=7.0$ and 8.0 are shown by magenta and green lines, respectively. c) The Baldwin-Phillips-Terlevich (BPT) diagram (Baldwin. Phillips \& Terlevich 1981). The dark-grey and light-grey dots are the same as in a). The green solid line by Kauffmann et al. (2003) separates AGN and SFGs. d) The WISE (W1 - W2) - (W2 - W3) colour-colour diagram, where $W 1, W 2, W 3$ are apparent magnitudes in the Vega system at $3.4 \mu \mathrm{m}, 4.6 \mu \mathrm{m}$ and $12 \mu \mathrm{m}$, respectively. SDSS SFGs are represented by dark-grey dots and SDSS QSOs (Schneider et al. 2010) by light-grey dots. HS $1851+6933$ is not shown because of the lack of WISE data. The meaning of other symbols in b) - d) is the same as in a). Two very low-metallicity galaxies with detected [Ne v] emission, SBS 0335-052E (blue-filled circle) and J1205+4551 (red-filled circle), are labelled in all panels.

\section{INTEGRATED PROPERTIES OF GALAXIES WITH [NE V] EMISSION}

Since the Izotov et al. (2012) study, we have found six more SFGs with detected [Nev] emission, increasing the number of such SFGs to fourteen and expanding further the range of oxygen abundances to higher values, up to $12+\log \mathrm{O} / \mathrm{H} \sim 7.9$. We have also extended that range to the lowest values by including one of the most metal-deficient galaxies known, J0811+4730, with $12+\log \mathrm{O} / \mathrm{H}=6.98$. Using the LBT observations of that galaxy by Izotov et al. (2018), we have estimated the $1 \sigma$ upper limit of its [Ne v] $\lambda 3426 / \mathrm{He}$ II $\lambda 4686$ flux ratio to be 0.15 . We have also included the extremely metal-deficient SFG J1631+4426 observed by Kojima et al. (2021). These authors provided intensities of emission lines including $\mathrm{He}$ II $\lambda 4686$, but not $[\mathrm{Ne} \mathrm{v}] \lambda 3426$.

The growing number of galaxies with detected [Nev] emission allows us to study better their properties and investigate possible mechanisms for generating hard ionizing radiation. For comparison, we use a sample of SFGs, some of which were observed in the wavelength range which includes the $[\mathrm{NeV}] \lambda 3426$ emission line. This sample was described in Sect.3 We wish to study similarities and differences in the integrated properties of SFGs with detected and nondetected $[\mathrm{Nev}] \lambda 3426$ emission. 


\subsection{Diagnostic diagrams}

Fig. 3a shows that all galaxies with [ $\mathrm{NeV}]$ emission are high-excitation objects that are located at the extreme end of the relation between $[\mathrm{O}$ III $] \lambda 5007 / \mathrm{H} \beta$ and $\mathrm{EW}(\mathrm{H} \beta)$ (Fig. 3a). This relation is a sort of evolutionary diagram, in the sense that the starburst age increases with both decreasing [O III] $\lambda 5007 / \mathrm{H} \beta$ and $\operatorname{EW}(\mathrm{H} \beta)$. Most of the galaxies with $[\mathrm{NeV}]$ emission follow well the sequence of SDSS galaxies at its upper end. However, there are three outliers. The first outlying galaxy with detected $[\mathrm{NeV}]$ emission, J1222+3602 (red star), likely a dwarf AGN, is offset to a higher $[\mathrm{O}$ III $] \lambda 5007 / \mathrm{H} \beta$ ratio, probably because of the presence of a non-thermal source. Two other galaxies, SBS 0335-052E (blue filled circle) and J1205+4551 (red filled circle), are offset from the sequence of compact SDSS SFGs (dark-grey dots) to lower $[\mathrm{O}$ III $] \lambda 5007 / \mathrm{H} \beta$ ratios because of their low metallicities, with $12+\log \mathrm{O} / \mathrm{H} \sim 7.3-7.4$.

For a fixed $\operatorname{EW}(\mathrm{H} \beta)$, there are a few other lowestmetallicity galaxies, but with non-detected [ $\mathrm{NeV}]$ emission, that are more offset from the sequence of compact SDSS SFGs, to lower values of $[\mathrm{O}$ III $] \lambda 5007 / \mathrm{H} \beta$. These galaxies are IZw $18 \mathrm{NW}$ and IZw $18 \mathrm{SE}$ (the two outermost black encircled filled circles), J1234+3901 and J2229+2725 (asterisks with lower and higher $\mathrm{EW}(\mathrm{H} \beta)$, respectively), and J1631+4426 (cyan encircled filled circle) with lower metallicities. The most deviant galaxy is J0811+4730 (magenta encircled filled circle) because of its extremely low metallicity.

There is a considerable number of other SFGs from the HeBCD sample (black symbols) which occupy the same region in Fig. 3 a as the SDSS SFGs and the most intense [Ne V] emitters, indicating similar excitation properties in their H II regions and metallicities. Some of these galaxies were observed in the wavelength range covering the $[\mathrm{Ne} \mathrm{V}] \lambda 3426$ emission line (black encircled filled circles). However, no [Nev] emission was detected, possibly due to the weakness of the line and/or an insufficient signal-to-noise ratio in the spectrum.

Fig. 3b shows that the galaxies with $[\mathrm{NeV}]$ emission are characterised by high $\mathrm{O}_{32}$ ratios. Three of them are among the objects with the highest known $\mathrm{O}_{32}$ ratios, $>20$. Most are offset from the relation defined by SDSS galaxies (black and grey dots), due to lower $R_{23}$ (= $([\mathrm{O}$ II $] \lambda 3727+[\mathrm{O}$ III $] \lambda 4959+[\mathrm{O}$ III $] \lambda 5007) / \mathrm{H} \beta))$ values, because of lower $12+\log \mathrm{O} / \mathrm{H}$, the most extreme offset being for SBS 0335-052E, with the lowest value $12+\log \mathrm{O} / \mathrm{H}=7.3$ (Thuan \& Izotov 2005) in the [Ne v] sample. For comparison, by magenta and green lines are shown loci of values modelled with the CLOUDY code for two oxygen abundances $12+\log \mathrm{O} / \mathrm{H}=7.0$ and 8.0 , respectively, and varying $\log$ of the ionization parameter $\log U$ in the range of -1.3 - 3.0. This offset is in accord with the analysis of Izotov et al. (2018) for the lowest-metallicity SFGs. In Fig.3b is shown for comparison the location of some lowest-metallicity galaxies with metallicities considerably lower than those in the galaxies with detected $[\mathrm{NeV}]$ emission, including the most deviant galaxy J0811+4730 (magenta encircled filled circle). This fact indicates that J0811+4730 has a lower metallicity than J1631+4426 (cyan encircled filled circle), contrary to the claim by Koiima et al. (2021).

It is seen in Fig. 3b that the galaxies with detected (blue and red symbols) and non-detected (open circles and some black encircled filled circles) $[\mathrm{Ne} \mathrm{v}] \lambda 3426$ emission line are overlapping, again indicating their similar properties, although the HeBCD galaxies (black encircled filled circles), which were selected for determination of the primordial He abundance, have systematically lower oxygen abundances.

A commonly used diagnostic diagram to distinguish between different sources of ionizing radiation in galaxies is the $[\mathrm{O}$ III $] \lambda 5007 / \mathrm{H} \beta-[\mathrm{N} \mathrm{II}] \lambda 6584 / \mathrm{H} \alpha$ diagram of Baldwin, Phillips \& Terlevich (BPT) Baldwin, Phillips \& Terlevich 1981), shown in Fig. 3r. We find that thirteen out of fourteen galaxies with $[\mathrm{Nev}] \lambda 3426$ emission are located in the region of star-forming galaxies, indicating that hard ionizing radiation is produced by the hottest stars or is a result of stellar evolution. The location of these galaxies in the diagram is similar to that of many galaxies with non-detected $[\mathrm{NeV}] \lambda 3426$ emission (black open circles and black encircled filled circles in Fig. 3 c). There is only one outlying galaxy, $\mathrm{J} 1222+3602$, where the source producing hard ionizing radiation is an AGN, according to the BPT diagram.

Another possible diagnostic diagram for distinguishing whether the ionizing radiation comes from stars or an AGN is the WISE colour - colour diagram (W1 - W2) $(W 2-W 3)$, where $W 1, W 2, W 3$ are mid-infrared WISE magnitudes at $3.4,4.6$, and $12 \mu \mathrm{m}$. In principle, emission of galaxies in the $W 3$ band is produced by warm dust, whereas in $W 1$ and $W 2$ bands it is of stellar and nebular origin. However, AGN can also heat dust to high temperatures of several hundred degree resulting in a high $W 1$ $W 2$ colour index. Thus, the location of a galaxy in the WISE colour diagram is determined by the properties of dust emission. In particular, the presence of hot dust with a temperature of several hundred $K$ will result in an increase of emission at $4.6 \mu \mathrm{m}$ and consequently with a large (red) $W 1-W 2$ colour index. In Fig. 3 d are shown SDSS SFG (dark-grey dots) and AGN (light-grey dots) points that are clearly separated, indicating the presence of hot dust in AGN. There is however an overlapping of AGN with a small fraction of SFGs characterized by $W 1-W 2 \gtrsim 0.5$ mag and $W 2-W 3 \gtrsim 3$ mag. Galaxies with $[\mathrm{Nev}] \lambda 3426$ emission are shown with large coloured symbols. Three of them, J0240-0828, J1205+4551 and J1222+3602, are located at the edge of the AGN region, where, however, many other SFGs are detected. Thus, the WISE colour - colour diagram in Fig. 3 $\mathrm{d}$ is not sufficient by itself to distinguish between stellar and nonthermal ionizing radiation. Furthermore, two galaxies in Fig.33 with [Nev] $\lambda 3426$ emission, $\mathrm{W} 1702+18$ and SBS 0335-052E, have $W 1-W 2>2.0 \mathrm{mag}$ and $W 2-W 3>4.0 \mathrm{mag}$, considerably higher than the corresponding colour indices for AGN (Griffith et al. 2011). Comparing with the galaxies from the comparison sample, we note that there is no evident difference in distributions of SFGs with detected and non-detected [Ne v] $\lambda 3426$ emission in Fig. 3 d.

We conclude from the above diagnostic diagrams that hard radiation produced by an AGN is likely present only in one galaxy, $\mathrm{J} 1222+3602$, whereas there is no evidence for such emission in the other thirteen galaxies. Overall, [Nev] $\lambda 3426$ emission is produced in high-excitation H II regions ionized by hot massive stars in most of the galaxies shown in Fig 3 by blue and red symbols. However, this emission is not seen in some other SFGs with similar excitation 


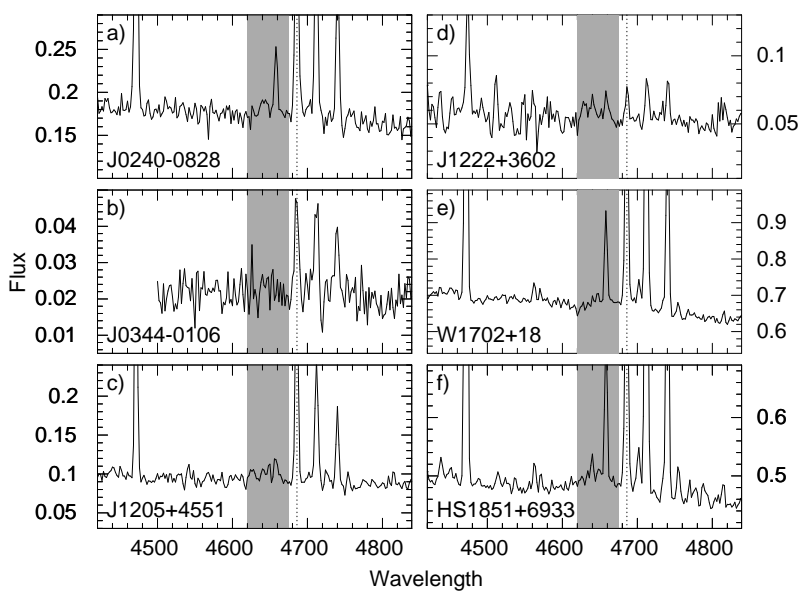

Figure 4. Segments of non-corrected for extinction rest-frame LBT spectra of new compact SFGs with a detected [Nev] $\lambda 3426$ emission line. The location of the He II $\lambda 4686$ emission line is indicated by a vertical dotted line. The location of broad Wolf-Rayet lines is shown by shaded regions.

conditions. Therefore, diagnostic diagrams are not sufficient by themselves for the selection of SFGs with [Ne v] $\lambda 3426$ emission.

\subsection{Evidence for stellar winds}

Wolf-Rayet (WR) stellar populations with ages $<4-5$ Myr have been suggested (e.g., Schaerer \& Stasińska 1999; Shirazi \& Brinchmann 2012) to provide hard ionizing radiation with photon energy $\geqslant 4$ ryd, and to be responsible for He II emission. WR broad emission at $\sim \lambda 4650$ (blue bump), which is a blend of stellar N III $\lambda 4634,4640$, C IV $\lambda 4658$ and HeII $\lambda 4686$ emission lines, and a broad C IV $\lambda 5808$ emission line (red bump) are frequently detected in spectra of star-forming galaxies with sufficiently high signal-to-noise ratio in the continuum (e.g., Guseva et al. 2000). However, the WR broad emission is rarely seen in nebular He IIemitting galaxies. Furthermore, Guseva et al. (2000) found that the nebular He II $\lambda 4686 / \mathrm{H} \beta$ ratio increases with decreasing metallicity, whereas the WR population decreases with decreasing metallicity. However, there are some exceptions, the most striking case being that of the SFG I Zw 18 with $12+\log \mathrm{O} / \mathrm{H} \sim 7.2$. Both a strong nebular He II $\lambda 4686$ emission line and broad blue and red WR bumps are seen in the spectrum with a high signal-to-noise ratio in the continuum of its northwest component (Izotov et al. 1997; Legrand et al. 1997).

We now check for the presence of WR features in the spectra of the new SFGs with $[\mathrm{NeV}] \lambda 3426$ emission. No broad C IV $\lambda 5808$ emission, the "red bump", a signature of the early carbon WR stars, has been detected in any of the galaxies. Additionally, no appreciable broad He II $\lambda 4686$ emission in the "blue bump" is seen. Segments of spectra that include regions of the WR blue bump are shown in Fig. 4 (shaded regions). It is seen that WR emission is present in the spectra of five out of the six studied galaxies, indicating the presence of late nitrogen WR stars. The exception is J0344-0106, where the signal-to-noise ratio in the continuum is too low to draw definite conclusions. The fluxes of the WR features were measured with the IRAF SPLOT routine and are shown in TableA1 after removal of the nebular [Fe III] $\lambda 4658$ emission line in the spectra of all galaxies, and of the narrow $\mathrm{N}$ III $\lambda 4640$ emission line in the spectrum of HS $1851+6933$. We note that a WR population is present even in the galaxy $\mathrm{J} 1205+4551$, with the lowest oxygen abundance $12+\log \mathrm{O} / \mathrm{H}=7.46$ in our sample of galaxies. Taking at face value the very high equivalent width of $519 \AA$ of its $\mathrm{H} \beta$ emission line, this indicates a very young starburst in $\mathrm{J} 1205+4551$, and that its broad WR emission is produced by the most massive stars, with masses of $\sim 100 \mathrm{M}_{\odot}$.

Two galaxies, J0240-0828 and Tol 1214-277, out of the fourteen galaxies with detected $[\mathrm{Nev}] \lambda 3426$ emission, have been observed with the HST in the far-UV range. A broad stellar Nv $\lambda 1240$ line with a P Cygni profile has been detected in the spectra of both galaxies (Thuan \& Izotov 1997; Jaskot et al. 2017). This implies that stellar winds, including those from WR stars, are at least present in some galaxies with $[\mathrm{Ne} \mathrm{V}] \lambda 3426$ emission.

However, WR emission is detected in only half of the fourteen galaxies with $[\mathrm{NeV}]$ emission discussed here, including galaxies in Thuan \& Izotov (2005) and Izotov et al. (2012). Furthermore, Izotov et al. (2012) modelled the intensity of nebular He II $\lambda 4686$ emission line and found that it is several times lower than the one observed even in the WR stage. Thus, we do not consider WR stars to be the main source of ionizing radiation in our sample.

\subsection{Evidence for fast gas motions}

Another possible source of hard ionizing radiation producing HeII $\lambda 4686$ and [ $\mathrm{NeV}] \lambda 3426$ emission is radiative shocks produced in the star-forming region by explosive processes like supernovae (SNe). Izotov et al. (2004a, 2012) have argued that, to explain the presence of both $[\mathrm{Ne} \mathrm{V}]$ and HeII emission, the propagation of shocks at a velocity of $300-500 \mathrm{~km} \mathrm{~s}^{-1}$ would be necessary. Observationally, these shocks should manifest themselves as the broad components of strong emission lines, such as the $\mathrm{H} \alpha$ emission line, formed in expanding envelopes and produced by multiple SN events.

We note that Izotov et al. (2004a, 2012) and Thuan \& Izotov (2005) observed galaxies with [Ne V] emission only in the blue range. On the other hand, the galaxies observed with the LBT/MODS presented here, have been observed both in the blue and red ranges. This allows us to search for low-intensity broad components of the $\mathrm{H} \alpha$ emission line. In Fig. [5 we show by a black dotted line the fit to the broad component of the $\mathrm{H} \alpha$ line for all galaxies in our sample.

Only the wings of the weak broad component are seen in the spectra, its central part being hidden by the very strong narrow $\mathrm{H} \alpha$ emission line and strong [N $\mathrm{II}]$ emission lines. Therefore, we derive the parameters of the broad component by using a single Gaussian to fit its wings. As an example, in Fig. 5r we show the profile of a comparison arc line (red dotted line), scaling its maximum profile intensity to the $\mathrm{H} \alpha$ maximum intensity. It is seen that the arc line is considerably narrower than the $\mathrm{H} \alpha$ line and shows much weaker broad wings, indicating that the stronger observed broad emission from the galaxies is not due to an instrumental effect, but real. We find that the velocity dispersions range from $\sim 750 \mathrm{~km} \mathrm{~s}^{-1}$ to $\sim 1240 \mathrm{~km} \mathrm{~s}^{-1}$. For all galax- 


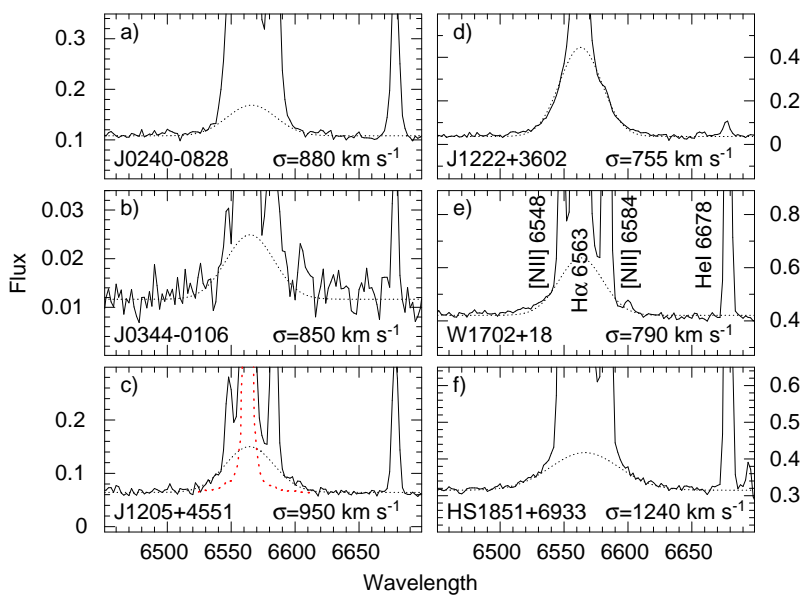

Figure 5. Parts of the rest-frame LBT spectra which include the $\mathrm{H} \alpha$ emission line of new compact SFGs with a detected $[\mathrm{NeV}] \lambda 3426$ emission line. Emission lines are labelled in panel e). The comparison arc emission line is shown for comparison in c) by the red dotted line. Its maximum intensity is scaled to be equal to the maximum intensity of the $\mathrm{H} \alpha$ emission line. Note the weak broad components of the $\mathrm{H} \alpha \lambda 6563$ emission line in all spectra. They are considerably broader than the arc line. Their fits by single Gaussians with labelled velocity dispersions $\sigma$ are shown by black dotted lines.

ies, excluding the dwarf AGN J1222+3602, the flux ratio of broad to narrow $\mathrm{H} \alpha$ components is a few per cent. On the other hand, this flux ratio is very high in $\mathrm{J} 1222+3602$. Izotov \& Thuan (2008) found it to be $\sim 72$ per cent, whereas the ratio derived from the LBT spectrum is $\sim 64$ per cent. The two observations having been made respectively in March 2005 and May 2015, the difference may be due to some small flux variability of the AGN.

No broad components of other emission lines have been detected in any galaxy, with the exception of J1222+3602. A broad $\mathrm{H} \beta$ component is detected in $\mathrm{J} 1222+3602$ with an intensity $\sim 10$ times lower than that of the broad $\mathrm{H} \alpha$ component and a velocity dispersion of $\sim 500 \mathrm{~km} \mathrm{~s}^{-1}$ or $2 / 3$ of the broad $\mathrm{H} \alpha$ velocity dispersion. A broad [O III] $\lambda 5007$ component is also detected in the spectrum of J1222+3602, with a $([\mathrm{O} \text { III }] \lambda 5007 / \mathrm{H} \beta)_{\text {broad }}$ flux ratio of $\sim 8.7$ and a velocity dispersion similar to that of the $\mathrm{H} \beta$ velocity dispersion. No other broad emission has been detected in this galaxy.

\section{MODELLING OF H II REGIONS WITH HARD IONIZING RADIATION}

In this section, we consider some mechanisms which can produce hard ionizing radiation in SFGs, and verify whether they are able to reproduce the observed intensities of the He II $\lambda 4686$ and [ $\mathrm{Ne} \mathrm{v}$ ] $\lambda 3426$ emission lines. For this purpose, we have calculated models of photoionized $\mathrm{H}$ II regions with the publicly available software code CLOUDY (Ferland et al. 2013), in combination with ionizing radiation derived from BPASS stellar population models alone (Eldridge et al. 2017), or from a combination of stellar radiation of STARBURST99 population synthesis models (Leitherer et al. 1999), with ionizing radiation produced by shocks (Allen et al. 2008) or by non-thermal radiation from an AGN. In these models, the lines of high-ionization species $\mathrm{He} I$ and $[\mathrm{Ne} \mathrm{V}]$ are due to the ionization by hard stellar or non-stellar ionizing radiation, whereas the lines of low-ionization species Hi, He I, [O II], [O III] etc are produced by softer stellar ionizing radiation. We aim to find models which satisfactorily reproduce the observed ratios of both the high- and low-ionization emission lines.

\subsection{Stellar radiation}

Hot massive stars are the main source of soft ionizing radiation in star-forming galaxies producing strong emission lines of H I, He I, [O II], [O III] and some other emission lines, mainly of singly and doubly ionized species. However, many studies have suggested that stellar emission can also be an important or even dominant source of hard ionizing radiation in low-metallicity galaxies, producing He II emission (e.g. Schaerer \& Stasińska 1999; Shirazi \& Brinchmann 2012; Kehrig et al. 2018; Kojima et al. 2021). On the other hand, only few studies on the role of stellar radiation in the origin of $[\mathrm{NeV}]$ emission have yet been carried out (Izotov et al. 2004a, 2012). In general, the origin of highionization species is not satisfactorily solved and the role of stellar radiation in producing both $\mathrm{He}$ II and [ $\mathrm{Ne} \mathrm{V}]$ emission remains unclear. In particular, Thuan \& Izotov (2005) have found from the STARBURST99 models that stellar radiation, including that of WR stars, is too soft for producing the observed He II $\lambda 4686$ emission in star-forming galaxies.

The situation is even worse for producing He II $\lambda 4686$ emission with fluxes above 2 per cent that of the $\mathrm{H} \beta$ emission line and detectable $\lceil\mathrm{NeV}\rceil \lambda 3426$ emission in low-metallicity SFGs (Table A1. Izotov et al. 2004a; Thuan \& Izotov 2005; Izotov et al. 2012). Unlike He II emission, ionizing radiation with energy above 7 Ryd is needed to produce [Ne v] $\lambda 3426$ emission. None of the STARBURST99 population synthesis models with stellar ionizing radiation are able to reproduce this emission at the detectable flux level of $\sim 0.1-1$ per cent that of the $\mathrm{H} \beta$ emission line.

Alternatively, BPASS population synthesis models can, possibly, account for the origin of hard ionizing radiation. These models include the evolution of close binaries producing hard radiation in accretion discs, capable of generating ions with high degrees of ionization. Below, we consider the BPASS stellar models including radiation from close binaries, and show that, contrary to expectations, these models are unable to reproduce the observed emission-line flux ratios and their equivalent widths in spectra of SFGs with [Nev] emission.

We use the CLOUDY v17.01 photoionization model calculations (Ferland et al. 1998, 2013), adopting the ionizing radiation from BPASS v2.1 population synthesis models, with the lowest heavy-element mass fraction $Z=$ $10^{-5}$, corresponding to the hardest available ionizing radiation. We also adopted the Salpeter IMF (Salpeter 1955) with a slope $\alpha=-2.35$, lower and upper mass limits $M_{\text {low }}=0.1 \mathrm{M}_{\odot}$ and $M_{\text {up }}=100 \mathrm{M}_{\odot}$, respectively. We note that adopting a top-heavy IMF with $\alpha=-2.00$ or an upper mass limit of $300 \mathrm{M}_{\odot}$ will only slightly increase the calculated emission-line fluxes. Varying the ISM oxygen abundances $12+\log \mathrm{O} / \mathrm{H}$ in the range $7.0-8.0$, we calculate the HeII $4686 / \mathrm{H} \beta,[\mathrm{NeV}] \lambda 3426 / \mathrm{He}$ II $\lambda 4686$ and [O III] $\lambda 5007 / \mathrm{H} \beta$ emission line ratios (Figs.6 -6 c) with the 

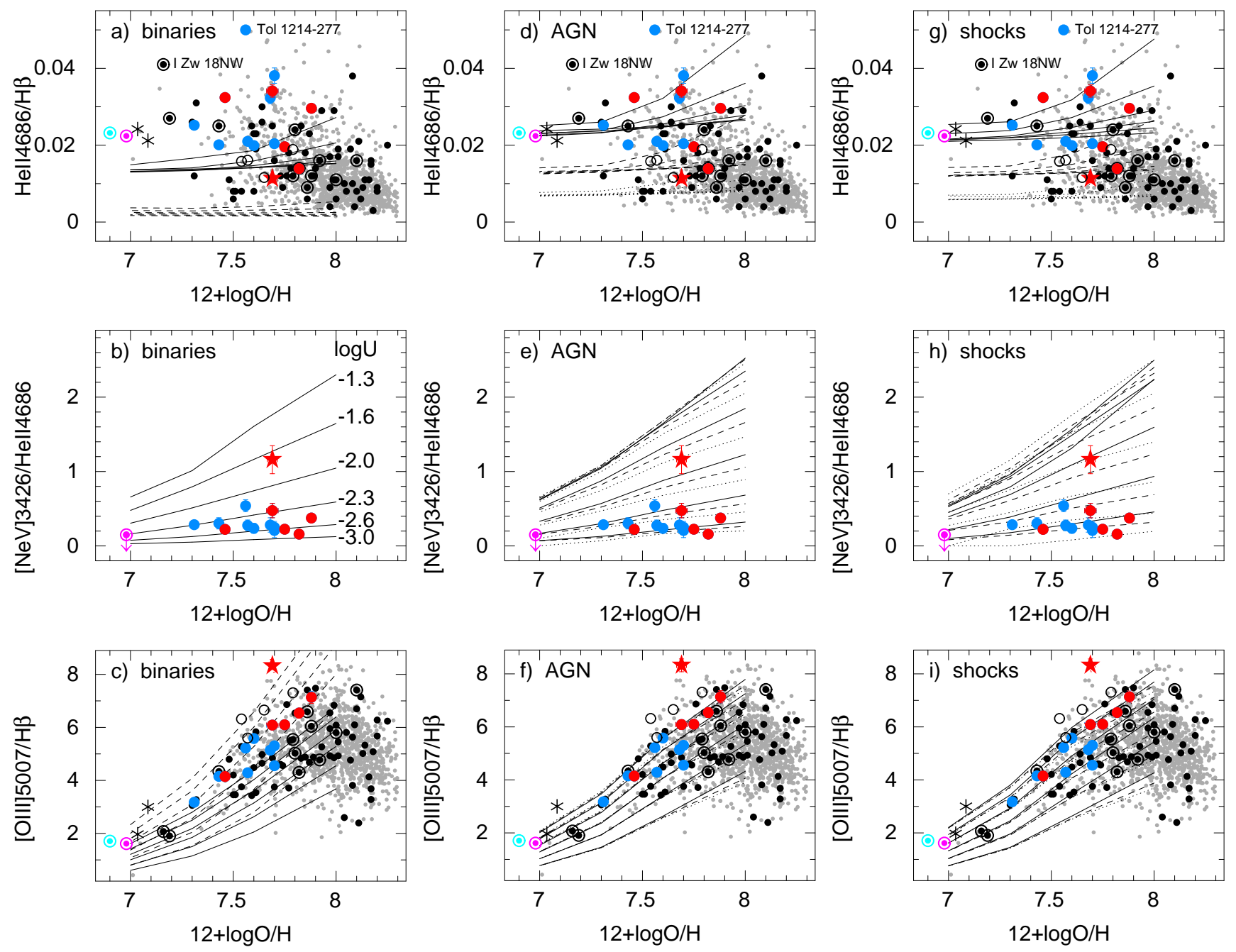

Figure 6. a) - c) Dependences of the He II $\lambda 4686 / \mathrm{H} \beta$, [Ne v] $\lambda 3426 / \mathrm{He}$ II $\lambda 4686$ and [O III] $\lambda 5007 / \mathrm{H} \beta$ emission-line ratios on the nebular oxygen abundance $12+\log \mathrm{O} / \mathrm{H}$ for BPASS models. Solid and dashed lines lines are for models with starburst ages of $20 \mathrm{Myr}$ and $2 \mathrm{Myr}$, respectively. Models are calculated with a stellar heavy element mass fraction of $10^{-5}$ and various ionization parameters $U$, labelled in b). d) - f) The same as in a) - c), but for STARBURST99 models including AGN and stellar ionizing radiation in proportions 5 and 95 per cent (dotted lines), 10 and 90 per cent (dashed lines) and in proportions 20 and 80 per cent (solid lines) for various ionization parameters $U \mathbf{. h})$ - i) The same as in a) - c), but for models including 90 per cent of stellar ionization radiation of STARBURST99 models and 10 per cent of shock ionizing radiation, with velocities $500 \mathrm{~km} \mathrm{~s}^{-1}$ (solid lines), $300 \mathrm{~km} \mathrm{~s}^{-1}$ (dashed lines) and $200 \mathrm{~km} \mathrm{~s}{ }^{-1}$ (dotted lines), for various ionization parameters. In all panels, the galaxies from this paper are shown with a red filled star (J1222+3602) and red filled circles (the five remaining galaxies). The galaxies with detected [Ne v] $\lambda 3426$ emission from Izotov et al. (2004a, 2012) and Thuan \& Izotov (2005) are shown with blue filled circles. Two galaxies with the He II $\lambda 4686 / \mathrm{H} \beta$ flux ratio above 4 per cent, Tol $1214-277$ and $\mathrm{IZw} 18 \mathrm{NW}$, are labelled in $\mathbf{a})$, d) and $\mathbf{g}$ ). Meaning of other symbols in all panels is the same as in Fig.2

IMF parameters $\alpha=-2.35, M_{\text {low }}=0.1 \mathrm{M}_{\odot}, M_{\text {up }}=100 \mathrm{M}_{\odot}$, for two starburst ages, $t=2 \mathrm{Myr}$ (dashed lines) and $t=$ $20 \mathrm{Myr}$ (solid lines) and various ionization parameters, labelled in Fig.6b.

It is seen in Fig.6 that only models with $t=20$ Myr are able to produce He II $\lambda 4686 / \mathrm{H} \beta$ ratios of $>1$ per cent, but even then, the predicted values are still lower than those observed in most galaxies with detected $[\mathrm{Ne} \mathrm{v}] \lambda 3426$ emission (red and blue symbols) and in most metal-deficient SFGs (cyan and magenta encircled filled circles, and asterisks). On other hand, they are similar to the observed intensities of the He II $\lambda 4686$ emission line in HeBCD galaxies (black filled circles), compact SFGs from the SDSS DR16 (grey dots) and a sample of SFGs with extreme $\mathrm{O}_{32}>20$ (black open circles), which show a decrease of the He II $\lambda 4686 / \mathrm{H} \beta$ flux ratio with increasing metallicity, confirming the finding by e.g. Guseva et al. (2000). The He II $\lambda 4686 / \mathrm{H} \beta$ ratio at larger ages (>20 Myr) quickly decreases to zero due to a softening of the ionizing radiation (Izotov et al. 2019). Correspondingly, it is unlikely to expect a detectable [ $\mathrm{Ne} \mathrm{V}]$ emission at these ages. We conclude that, with the higher observed values of the He II $\lambda 4686 / \mathrm{H} \beta$ ratio, stellar ionizing radiation is unlikely to account for He II emission in most SFGs with low oxygen abundances $12+\log \mathrm{O} / \mathrm{H} \lesssim 7.7$, including nearly all galaxies with detected [ $\mathrm{Ne} \mathrm{V}]$ emission (Fig.66). In the same manner, CLOUDY models in combination with BPASS v2.1 population synthesis models including only single stars, and with STARBURST99 population synthesis models also predict 
a very low He II $\lambda 4686$ line intensity, $\lesssim 0.1$ per cent that of $\mathrm{H} \beta$.

In Fig.6b, we show the dependence of the [Nev] $\lambda 3426 / \mathrm{He}$ II $\lambda 4686$ flux ratio on oxygen abundance $12+\log \mathrm{O} / \mathrm{H}$ for the same SFGs as in Fig.6 6 , but with detected $[\mathrm{NeV}] \lambda 3426$ emission. The solid lines show CLOUDY model predictions with ionizing radiation of the BPASS model with $Z=10^{-5}$ and $t=20 \mathrm{Myr}$, for various ionization parameters $U$. We do not show models with $t=2 \mathrm{Myr}$, because they do not produce any $[\mathrm{NeV}] \lambda 3426$ emission. The increase of the modelled [Ne v] $\lambda 3426 / \mathrm{He}$ II $\lambda 4686$ flux ratio with increasing $12+\log \mathrm{O} / \mathrm{H}$, at a fixed $U$, is caused in part by the increased $\mathrm{Ne}$ abundance. At variance with the models, the observed ratios are nearly independent of $12+\log \mathrm{O} / \mathrm{H}$, with $\log U$ between -3.0 and -2.3 . However, the assumption of an extremely low stellar heavy element mass fraction, $Z=10^{-5}$, is not reasonable for SFGs with much higher observed nebular $12+\log \mathrm{O} / \mathrm{H}$ (between 7.46 and 7.88 or $1 / 17$ and $1 / 7$ solar, adopting $12+\log \mathrm{O} / \mathrm{H}=8.7$ for the Sun). The models with higher stellar heavy element mass fraction, $Z=10^{-3}$, corresponding to the observed nebular oxygen abundances, would result in several times lower fluxes of $\mathrm{He}$ II and [ $\mathrm{Ne} \mathrm{V}]$. In summary, we conclude that stellar radiation is unlikely to be the source of hard ionizing radiation responsible for the high ionization lines.

The relation between the [O III] $\lambda 5007 / \mathrm{H} \beta$ emission line ratios and oxygen abundances are shown in Fig.6r. It is seen that only models with $t=2$ Myr can reproduce the observations of the galaxies with detected [Ne v] $\lambda 3426$ emission. Furthermore, the observed equivalent widths of the $\mathrm{H} \beta$ emission line in the galaxies with detected $[\mathrm{Ne} \mathrm{V}] \lambda 3426$ emission are very high (Table A1) and are consistent with the $t=2 \mathrm{Myr}$ instantaneous burst model, but not with the $t=20$ Myr instantaneous burst model, characterised by $\mathrm{EW}(\mathrm{H} \beta) \sim 10-20 \AA$. The highest $\operatorname{EWs}(\mathrm{H} \beta)$ of $\sim 300-500 \AA$ in some of our galaxies can not also be explained by continuous star formation.

These comparisons between observations and models support the overall conclusion that stellar ionizing radiation, calculated from both STARBURST99 and BPASS models, fails to reproduce simultaneously the observed emission line fluxes in galaxies with a detected $[\mathrm{Nev}] \lambda 3426$ emission line. The instantaneous burst models with $t=20 \mathrm{Myr}$ predict too low line intensities of high-ionized (He II $\lambda 4686$ ) and low-ionized ( [O III $] \lambda 5007)$ species in these galaxies compared to observations (red and blue symbols in Fig.6 - 6 - r). However, the observed properties of most less extreme HeBCD galaxies (black filled and encircled filled circles), SFGs with extreme $\mathrm{O}_{32}>20$ (black open circles) and compact SDSS DR16 galaxies (grey dots) with higher metallicities can be reproduced by the BPASs models with burst ages between 2 and $20 \mathrm{Myr}$.

\subsection{AGN}

Strong [Ne V] $\lambda 3426$ and He II $\lambda 4686$ emission lines are commonly observed in high-excitation Sy2 galaxies, with typical $[\mathrm{Ne} \mathrm{V}] \lambda 3426 / \mathrm{He}$ II $\lambda 4686$ flux ratios of $\sim 2-3$. These values can be obtained in an $\mathrm{H}$ II region with solar metallicity, ionized by a non-thermal source with a power-law distribution of ionizing radiation (e.g. Izotov et al. 2004a).

The dominant source in our galaxies is softer stel- lar emission, with effective temperatures of the most massive main-sequence stars $\lesssim 55000 \mathrm{~K}$, which make it unable to excite detectable [Nev] and HeII emission. Considerably higher effective temperatures, $\gtrsim 100000 \mathrm{~K}$, are needed. These high temperatures can be found in hot white dwarfs and early-type WR stars. However, luminosities of white dwarfs are low, so that they are unable to produce large amount of ionizing photons. Furthermore, emission lines in our SFGs are produced by young star-forming regions, whereas white dwarfs appear after $100 \mathrm{Myr}$. On the other hand, WR stellar populations are also unable to produce He II $\lambda 4686$ emission line with the observed intensities. Therefore, an another additional source of hard ionizing radiation is needed. We consider here that additional source to be nonthermal power-law radiation produced by an AGN.

Izotov et al. (2012) have shown that to account for the observed strengths of both the [Ne v] $\lambda 3426$ and He II $\lambda 4686$ emission lines, simultaneously with that of the [O III $] \lambda 5007$ line in the galaxies with detected $[\mathrm{NeV}]$ emission, the number of the AGN ionizing photons should be $\sim 10$ per cent of the number of softer stellar ionizing photons. Furthermore, the ISM oxygen abundances in our galaxies and the corresponding Ne abundances are about one order of magnitude lower than typical values for Sy2 galaxies. Therefore, if non-thermal sources of ionization are present in our SFGs, the $[\mathrm{Ne} \mathrm{V}] \lambda 3426 / \mathrm{He}$ II $\lambda 4686$ flux ratio is expected to be lower than in Sy2 galaxies because of their lower Ne abundance. This reduced ratio is indeed observed in SFGs with $[\mathrm{Ne} \mathrm{v}] \lambda 3426$ emission.

Figs.61-6f display the same diagrams as in Figs.676r, but with population synthesis models combining the STARBURST99 stellar models with an AGN source of nonthermal radiation, the latter contributing 5 per cent (dotted lines), 10 per cent (dashed lines) and 20 per cent (solid lines) of the total number of ionizing photons. The extreme-UV and X-ray part of this AGN radiation, generating He II and $[\mathrm{Ne} \mathrm{v}]$ emission, is represented by a power-law (Ferland et al. 2013). Fig.6 6hows that the He II $\lambda 4686 / \mathrm{H} \beta$ flux ratio in galaxies with detected $[\mathrm{NeV}] \lambda 3426$ emission can be reproduced by models mainly with 20 per cent of AGN contribution. However, the ratios in some galaxies can be modelled with 5 or 10 per cent of AGN contribution. There is one outlying galaxy with a detected [Ne v] $\lambda 3426$ emission line, Tol 1214-277. It has a strong He II $\lambda 4686$ emission line, with a flux equal to 5 per cent that of $\mathrm{H} \beta$, that can not be reproduced by any model. Another object, I Zw $18 \mathrm{NW}$, with a He II $\lambda 4686$ emission line flux of $\sim 4$ per cent that of the $\mathrm{H} \beta$ emission line is located in a region not covered by any model. However, no [ $\mathrm{NeV}$ ] emission is detected in I Zw $18 \mathrm{NW}$. On the other hand, the He II $\lambda 4686$ emission line is observed in most of the galaxies with a high $\mathrm{O}_{32}$ from the HeBCD and SDSS samples (black open circles, black filled circles and grey dots, respectively). It can be reproduced by models with AGN contributions between 5 and 20 per cent.

The $[\mathrm{Nev}] \lambda 3426 / \mathrm{He}$ II $\lambda 4686$ and $[\mathrm{O}$ III $] \lambda 5007 / \mathrm{H} \beta$ emission line ratios are shown in Figs.6e and 6f, respectively. It is seen that, with the exception of one galaxy, the models reproduce well the observations. The exception is the dwarf AGN galaxy J1222+3602, where emission from highionization species is formed in the narrow line region (NLR) of the AGN. The observed [O III] $\lambda 5007 / \mathrm{H} \beta$ emission line flux ratio in this galaxy is considerably higher than the range of 
values predicted by the models (Fig.6f). In principle, increasing the fraction of ionizing photons due to the powerlaw source would increase the modelled ratio. However, this would unacceptably increase the predicted $\mathrm{He}$ II $\lambda 4686 / \mathrm{H} \beta$ flux ratio, compared to the observed one. What causes this difference between observations and models is not clear.

All our galaxies were not detected in the X-ray range by the Roentgen Satellite (ROSAT). We also searched in the Chandra source catalogue. Only J1222+3602 has been detected, supporting the conclusion that [Ne v] $\lambda 3426$ and He II $\lambda 4686$ emission is mainly powered in this galaxy by an AGN source, with a power-law ionizing emission extending to the X-ray range. All other galaxies are likely powered by the ionizing emission of stellar populations and radiative shocks. We note that even the bright blue compact dwarf (BCD) star-forming galaxy Tol 1214-277, with the highest $[\mathrm{Ne} \mathrm{v}] \lambda 3426 / \mathrm{H} \beta$ flux ratio known (Izotov et al. 2004a), was not detected by either ROSAT or Chandra.

\subsection{Radiative shocks}

Izotov et al. (2012) have suggested that interstellar radiative shocks with velocities $v_{s}$ of $300-500 \mathrm{~km} \mathrm{~s}^{-1}$ can also produce enough extreme UV radiation to account for the observed He II $\lambda 4686 / \mathrm{H} \beta$ emission-line ratios of $\sim 2-3$ per cent, and for the $[\mathrm{Ne} \mathrm{V}] \lambda 3426 / \mathrm{He}$ II $\lambda 4686$ emission line ratios of $\sim 0.2-0.5$ measured in the present sample of galaxies. The dependence of this ratio on $v_{s}$ is complex, with both shock and precursor components having to be taken into account (Fig. 18 and Table 6 in Allen et al. 2008). The fraction of shock radiation in the $\mathrm{H}$ II region should be small compared to the fraction of softer stellar ionizing radiation in order to reproduce both the high- and lower-ionization emission lines. Shock radiation plays the main role in the production of He II $\lambda 4686$ and [Ne V] $\lambda 3426$ emission, whereas the softer stellar radiation is mainly responsible for the existence of lower-ionization emission lines such as Hi, HeI, [O II], [O III] etc. The dominance of stellar ionizing radiation is also required to reproduce the high observed $\mathrm{O}_{32}$ ratios, because pure shock models predict very low $\mathrm{O}_{32}, \lesssim 0.5$. This is similar to the low $\mathrm{O}_{32}$ predicted in pure AGN models.

To further investigate the role of radiative shocks in producing extreme-UV and X-ray emission, we consider a set of CLOUDY uniform and spherically-symmetric composite models with ionizing radiation consisting of two components. The first component is the radiation calculated with the STARBURST99 code, of a single stellar population with an age of $2 \mathrm{Myr}$, a production rate of ionizing photons above 1 ryd $Q_{\text {stellar }}=10^{53} \mathrm{~s}^{-1}$ (this corresponds to the average $\mathrm{H} \beta$ luminosity of the studied galaxies) and various metallicities. The second component is the radiation from radiative shocks calculated by Allen et al. (2008), coincident with the source of stellar radiation, and characterized by the three shock velocities of 200,300 and $500 \mathrm{~km} \mathrm{~s}^{-1}$. We adopt the production rate of ionizing photons above 1 ryd, $Q_{\text {shock, }}$, to

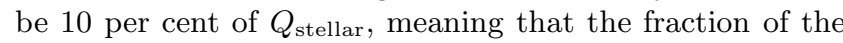
$\mathrm{H} \beta$ luminosity due to shocks is set to be equal to 10 per cent of the $\mathrm{H} \beta$ luminosity produced by stellar ionizing radiation.

We calculate models varying the filling factor in a wide range so that the log of the ionization parameter $U$, averaged over the $\mathrm{H}$ II region volume, is in the range $-1.3--3.0$. The same range of $\log U$ was adopted for models with pure stellar emission and for models with a mixture of stellar and non-thermal AGN emission (Sections 5.1 and 5.2).

In Figs. 6s - 6, we show the dependences on oxygen abundance of the emission-line ratios discussed above, for composite models with shock velocities of $500 \mathrm{~km} \mathrm{~s}^{-1}$ (solid lines), $300 \mathrm{~km} \mathrm{~s}^{-1}$ (dashed lines) and $200 \mathrm{~km} \mathrm{~s}^{-1}$ (dotted lines), and with $Q_{\text {shock }} / Q_{\text {stellar }}=10$ per cent. In general, composite models with shock velocities of $500 \mathrm{~km} \mathrm{~s}^{-1}$ reproduce well the observed emission-line ratios in galaxies with detected $[\mathrm{Ne} \mathrm{v}] \lambda 3426$ emission (red and blue symbols), as is the case of models with power-law AGN ionizing spectra with 20 per cent of the radiation produced by an AGN (Figs.6d - 6f). Correspondingly, the He II $\lambda 4686$ emissionline intensities in galaxies from the HeBCD and SDSS samples can better be reproduced by models with shock velocities between $200 \mathrm{~km} \mathrm{~s}^{-1}$ and $300 \mathrm{~km} \mathrm{~s}^{-1}$. Alternatively, the observed He II $\lambda 4686$ emission-line intensities can also be reproduced by models in which the fraction of ionizing photons produced by a shock, $Q_{\text {shock }} / Q_{\text {stellar }}$, is varied, instead of the shock velocity $v_{s}$.

On the other hand, given a fixed oxygen abundance, the $[\mathrm{NeV}] \lambda 3426 / \mathrm{He}$ II $\lambda 4686$ ratio only weakly depends on variations of the fractions of the AGN or shock ionizing radiation in the ranges considered in this paper, so that the $[\mathrm{NeV}] \lambda 3426$ emission-line intensity can be estimated from the He II $\lambda 4686$ emission-line intensity and its detection in star-forming galaxies depends mainly on the signal-to-noise ratio of the spectrum. Practically, $[\mathrm{NeV}] \lambda 3426$ emission is detected in SFGs with a He II $\lambda 4686 / \mathrm{H} \beta$ line ratio $\gtrsim 2$ per cent (this paper, Izotov et al. 2004a, 2012).

The reason for the similarity of the two types of models, with AGN and shock emission, is that the ratio of the numbers of photons $Q$ (HeII) with energy $h \nu>54 \mathrm{eV}$ ionizing HeII, to $Q(\mathrm{HI})$ with energy $13.6 \mathrm{eV}<h \nu<24 \mathrm{eV}$ ionizing $\mathrm{HI}$, rapidly decreases with decreasing shock velocity $v_{s}$, in the range $v_{s} \lesssim 300 \mathrm{~km} \mathrm{~s}^{-1}$. However, that ratio remains fairly constant at a high value, $Q(\mathrm{He} \mathrm{II}) / Q(\mathrm{HI}) \sim$ $0.6-0.7$, in the velocity range $v_{s} \gtrsim 500 \mathrm{~km} \mathrm{~s}^{-1}$. The spectral energy distribution for $h \nu>24 \mathrm{eV}$, including the photon energies above $97 \mathrm{eV}$ responsible for [ $\mathrm{Ne} \mathrm{v}$ ] emission, can be represented by a power law with a slope $\alpha \sim-1$, similar to the typical X-ray spectrum of an AGN (Ferland et al. 2013).

The common features which both AGN and shock models share are the following: 1) the He II $\lambda 4686 / \mathrm{H} \beta$ emissionline ratios depend weakly on the ionization parameter, 2) the $[\mathrm{Ne} \mathrm{V}] \lambda 3426 / \mathrm{He}$ II $\lambda 4686$ emission line ratios can be reproduced only by models with low ionization parameters and 3 ) the $[\mathrm{O}$ III $] \lambda 5007 / \mathrm{H} \beta$ emission line ratios can be reproduced only by models with high ionization parameters. Thus, uniform HII region models, with a constant density, likely cannot account simultaneously for all observed emission-line ratios. More plausible are non-uniform models with low-density channels produced by outflows, where He II and $[\mathrm{Ne} \mathrm{V}]$ are emitted via illumination by harder ionizing radiation of radiative shocks propagating through these channels, whereas [O III] emission originates in denser regions exposed to softer stellar ionizing sources.

Finally, we note that the He II $\lambda 4686 / \mathrm{H} \beta$ flux ratios above 4 per cent in two BCDs, Tol 1214-277 and I Zw $18 \mathrm{NW}$, are not reproduced by any model shown in Fig.6 These unusually high values can, in principle, be explained if the HeII $\lambda 4686$ line is emitted in a density 
bounded H II region or in low-density holes produced by outflows. This case is likely applicable to Tol 1244-277 and to another SFG, J0240-0828, with [NeV] emission and a He II $\lambda 4686 / \mathrm{H} \beta$ flux ratio about 3 per cent. The strong Ly $\alpha$ emission line observed in the UV spectra of Tol 1244-277 and J0240-0828 (Thuan \& Izotov 1997; Jaskot et al. 2017) possibly indicates escape of ionizing radiation through lowdensity channels. In J0240-0828, the velocity separation between the blue and red peaks of the Ly $\alpha$ emission-line profile is small, $\sim 266 \mathrm{~km} \mathrm{~s}^{-1}$ (Jaskot et al. 2017), whereas strong emission at the center of the Ly $\alpha$ line is present in the spectrum of Tol 1244-277 (Thuan \& Izotov 1997). The UV spectra of both objects indicate a low optical depth of the neutral gas. However, the assumption of a density bounded H II region does not apply to I Zw $18 \mathrm{NW}$, because of the high optical depth of neutral gas around this galaxy, as indicated by a strong absorption Ly $\alpha$ line (Kunth et al. 1994). Alternatively, Kehrig et al. (2015, 2021) have analysed the possible role of X-ray ionizing radiation from the high-mass X-ray binary (HMXB) in I Zw $18 \mathrm{NW}$, in generating strong He II $\lambda 4686$ emission. They found that the X-ray luminosity of the HMXB is not large enough to reproduce the observed intensity of this line. Thus, the presence of strong HeII emission and the absence of [Ne V] emission in I Zw $18 \mathrm{NW}$ remains puzzling and can not be reproduced by any of the considered models.

\section{CONCLUSIONS}

In this paper, we present Large Binocular Telescope (LBT) spectrophotometric observations of a sample of six compact star-forming galaxies (SFG) with detected high-ionization He II $\lambda 4686$ and [Nev] $\lambda 3426$ emission lines. All selected galaxies have been observed in the optical range with the Multi-Object Dual Spectrograph (MODS) and four of them have also been observed with the LBT Utility Camera in the Infrared (LUCI) spectrograph, in the near-infrared $z$ and $J$ bands. Our main results are as follows.

1. The He II $\lambda 4686$ emission line (ionization potential $\sim$ $54 \mathrm{eV}$ ) with fluxes of $\sim 1-3.5$ per cent that of the $\mathrm{H} \beta$ emission line, the $[\mathrm{Ne} \mathrm{v}] \lambda 3426$ emission line (ionization potential $\sim 97 \mathrm{eV}$ ) with fluxes of $\sim 0.2-1.6$ per cent that of the $\mathrm{H} \beta$ flux, and the $[\mathrm{FeV}] \lambda 4227$ emission line (ionization potential $\sim 54.8 \mathrm{eV})$ with fluxes of $\sim 0.3-0.7$ per cent that of the $\mathrm{H} \beta$ flux (excluding $\mathrm{J} 1222+3602$ which is likely a dwarf AGN) are detected in our sample of galaxies. The $[\mathrm{NeV}] \lambda 3426 / \mathrm{He}$ II $\lambda 4686$ flux ratio varies in the range from $\sim 0.2$ to $\sim 0.4$ in five galaxies, and attaining the highest value of $\sim 1.2$ in $\mathrm{J} 1222+3602$. These ratios are similar to those obtained for the eight galaxies with He II $\lambda 4686$ and $[\mathrm{Nev}] \lambda 3426$ emission discussed previously by Izotov et al. (2004a, 2012) and Thuan \& Izotov (2005). However, the ratio is considerably higher in J1222+3602, likely due to the presence of an AGN and emission of both HeII and [Ne V] in its narrow-line region.

2. All sample galaxies are characterised by low oxygen abundances $12+\log \mathrm{O} / \mathrm{H}=7.46-7.88$, very high equivalent widths $\operatorname{EW}(\mathrm{H} \beta) \sim 190-520 \AA$. Very dense $\mathrm{H}$ II regions with high electron number densities of $\sim 300-700 \mathrm{~cm}^{-3}$ are observed in five out of six SFGs. These electron densities are derived from the $[\mathrm{S} \mathrm{II}] \lambda 6717 / \lambda 6731$ and/or
He I $\lambda 10831 / \lambda 6678$ emission-line ratios. The signal-to-noise ratio of the MODS spectrum of J0344-0106 is too low to definitely derive $N_{\mathrm{e}}(\mathrm{S} I \mathrm{I})$, and no LUCI observations are available for this galaxy to derive $N_{\mathrm{e}}$ (He I). All studied galaxies are also characterised by very high $\mathrm{O}_{32}=$ [O III $] \lambda 5007 /[\mathrm{O}$ II $] \lambda 3727$ ratios, in the range $10-30$, indicating a high degree of ionization.

3. Five galaxies are located in the upper part of the SFG branch in the BPT diagram, indicating ionization by stellar emission. Only H II regions in J1222+3602 are likely, at least in part, powered by AGN ionizing radiation. The [O III $] \lambda 5007 / \mathrm{H} \beta$ ratio ranges from $\sim 4$ in the spectrum of the lowest-metallicity galaxy $\mathrm{J} 1205+4551$, to $\sim 8$ in the spectrum of J1222+3602, characteristic of high-excitation H II regions. Wolf-Rayet broad emission is likely present in five galaxies. The signal-to-noise ratio of the J0344-0106 spectrum is too low to be sure of the presence of broad WR emission. Broad low-intensity components of the $\mathrm{H} \alpha$ emission line are present in the spectra of all six galaxies. The flux ratios of the broad-to-narrow $\mathrm{H} \alpha$ components are a few per cent in five galaxies, but goes up to $\sim 70$ per cent in $\mathrm{J} 1222+3602$.

4. We discuss possible sources of hard radiation producing HeII and [Nev] emission. The main conclusion is that pure stellar ionizing radiation is unlikely to do the job. Comparing the model predictions and observational data for a sample of fourteen galaxies with simultaneously detected high-ionization lines He II $\lambda 4686$ and [Ne v] $\lambda 3426$ (there are six new such galaxies in this paper), we conclude that the most likely source of hard radiation is fast radiative shocks, with velocities of $\sim 500 \mathrm{~km} \mathrm{~s}^{-1}$, which are natural outcome of massive star evolution through supernovae explosions in young star-forming regions. For the present sample, shock ionizing radiation in five galaxies and AGN emission in the sixth one, J1222+3602, can succesfully reproduce the observed fluxes of high-ionization lines. However, observed emission-line ratios are also consistent with the presence an AGN source, contributing $\sim 10$ per cent to the total luminosity of ionizing radiation, in all considered galaxies, in addition to the one in $\mathrm{J} 1222+3602$.

In both AGN and shock models, the $[\mathrm{NeV}] \lambda 3426$ /He II $\lambda 4686$ emission line ratios can only be reproduced by models with low ionization parameters while the [O III $] \lambda 5007 / \mathrm{H} \beta$ emission line ratios can be reproduced only by models with high ionization parameters. Thus, most plausible are non-uniform models, where He II and [Nev] lines are emitted in low-density channels produced by outflows and illuminated by harder ionizing radiation from radiative shocks propagating through these channels, whereas [O III] emission originates in denser regions exposed to softer stellar ionizing sources.

\section{ACKNOWLEDGEMENTS}

Y.I. and N.G. acknowledge support from the National Academy of Sciences of Ukraine by its priority project No. 0120U100935 "Fundamental properties of the matter in the relativistic collisions of nuclei and in the early Universe". Funding for the Sloan Digital Sky Survey IV has been provided by the Alfred P. Sloan Foundation, the U.S. Department of Energy Office of Science, and the Participating Insti- 
tutions. SDSS-IV acknowledges support and resources from the Center for High-Performance Computing at the University of Utah. The SDSS web site is www.sdss.org. SDSS-IV is managed by the Astrophysical Research Consortium for the Participating Institutions of the SDSS Collaboration. This research has made use of the NASA/IPAC Extragalactic Database (NED), which is operated by the Jet Propulsion Laboratory, California Institute of Technology, under contract with the National Aeronautics and Space Administration.

\section{DATA AVAILABILITY}

The data underlying this article will be shared on reasonable request to the corresponding author.

\section{REFERENCES}

Allen M. G., Groves B. A., Dopita M. A., Sutherland R. S., Kewley L. J., 2008, ApJS, 178, 20

Baldwin J. A., Phillips M. M., Terlevich R., 1981, PASP, 93,5

Dopita M. A., Sutherland R. S., 1996, ApJS, 102, 161

Eldridge J. J., Stanway E. R., Xiao L., McClelland L. A. S., Taylor G., Ng M., Greis S. M. L., Bray J. C., 2017, PASA, 34, 58

Ferland G. J., Korista K. T., Verner D. A., Ferguson J. W., Kingdon J. B., Verner E. M., 1998, PASP, 110, 761

Ferland G. J. et al., 2013, Revista Mexicana de Astronomia y Astrofisica, 49, 137

Filippenko A. V., 1982, PASP, 94, 715

Fricke K. J. et al., 2001, AJ, 121, 169

Garnett D. R., Kennicutt Jr. R. C., Chu Y.-H., Skillman E. D., 1991, ApJ, 373, 458

Griffith R. L. et al., 2011, ApJ, 736, L22

Guseva N. G., Izotov Y. I., Thuan T. X., 2000, ApJ, 531, 776

Izotov Y. I., Thuan T. X., 2004, ApJ, 602, 200

Izotov Y. I., Thuan T. X., 2008, ApJ, 687, 133

Izotov Y. I., Thuan T. X., Lipovetsky V. A., 1994, ApJ, 435,647

Izotov Y. I., Foltz C. B., Green R. F., Guseva N. G., Thuan T. X., 1997, ApJ, 487, L37

Izotov Y. I., Chaffee F. H., Schaerer D., 2001, A\&A, 378, L45

Izotov Y. I., Noeske K. G., Guseva N. G., Papaderos P., Thuan T. X., Fricke K. J., 2004a, A\&A, 415, L27

Izotov Y. I., Stasińska G., Guseva N. G., Thuan T. X., 2004a, A\&A, 415, 87

Izotov Y. I., Stasińska G., Meynet G., Guseva N. G., Thuan T. X., 2006a, A\&A, 448, 955

Izotov Y. I., Schaerer D., Blecha A., Royer F., Guseva N. G., North P., 2006b, A\&A, 459, 71

Izotov Y. I., Thuan T. X., Guseva N. G., 2007, ApJ, 671, 1297

Izotov Y. I., Thuan T. X., Privon G., 2012, MNRAS, 427, 1229

Izotov Y. I., Stasińska G., Guseva N. G., 2013, A\&A, 558, A57
Izotov Y. I., Thuan T. X., Guseva N. G., 2014, MNRAS, 445,778

Izotov Y. I., Thuan T. X., Guseva N. G., 2017, MNRAS, 471,548

Izotov Y. I., Thuan T. X., Guseva N. G., Liss S. E., 2018, MNRAS, 473, 1956

Izotov Y. I., Thuan T. X., Guseva N. G., 2019, MNRAS, 483, 5491

Izotov Y. I., Thuan T. X., Guseva N. G., 2021, MNRAS, 504, 3996

Jaskot A. E., Oey M. S., Scarlata C., Dowd T., 2017, ApJ, 851, L9

Kauffmann G. et al., 2003, MNRAS, 341, 33

Kehrig C., Vílchez J. M., Pérez-Montero E., IglesiasPáramo J., Brinchmann J., Kunth D., Durret F., Bayo F. M., 2015, ApJ, 801, L28

Kehrig C., Vílchez J. M., Guerrero M. A., Iglesias-Páramo J., Hunt L. K., Duarte-Puertas S., Ramos-Larios G., 2018, MNRAS, 480, 1081

Kehrig C., Guerrero M. A., Vílchez J. M., Ramos-Larios G., 2021, ApJ, 908, 54

Kojima T. et al., 2021, ApJ, 913, 22

Kunth D. Lequeux J., Sargent W. L. W., Viallefond F., 1994, A\&A, 282, 709

Legrand F., Kunth D., Roy J.-R., Mas-Hesse J. M., Walsh J. R., 1997, A\&A, 326, L17

Leitherer C. et al., 1999, ApJS, 123, 3

Leitherer C., Ekström S., Meynet G., Schaerer D., Agienko

K. B., Levesque E. M., 2014, ApJS, 212, 14

Pakull M. W., Angebault L. P., 1986, Nature, 322, 511

Porter R. L., Ferland G. J., Storey P. J., Detisch M. J., 2013, MNRAS, 433, L89

Salpeter E. E., 1955, ApJ, 121, 161

Schaerer D., 1996, ApJ, 467, L17

Schaerer D., Fragos T., Izotov Y. I., 2019, A\&A, 622,L10

Schaerer D., Stasińska G., 1999, A\&A, 345, L17

Schneider D. P., Richards G. T., Hall P. B. et al., 2010, AJ, 139, 2360

Shirazi M., Brinchmann J., 2012, MNRAS, 421, 1043

Thuan T. X., Izotov Y. I., 1997, ApJ, ApJ, 489, 623

Thuan T. X., Izotov Y. I., 2005, ApJ, ApJS, 161, 240

Wright E. L., 2006, PASP, 118, 1711

\section{APPENDIX A: EMISSION-LINE FLUXES AND ELEMENT ABUNDANCES}

This paper has been typeset from a $\mathrm{T}_{\mathrm{E}} \mathrm{X} / \mathrm{L}_{\mathrm{E}} \mathrm{X}$ file prepared by the author. 

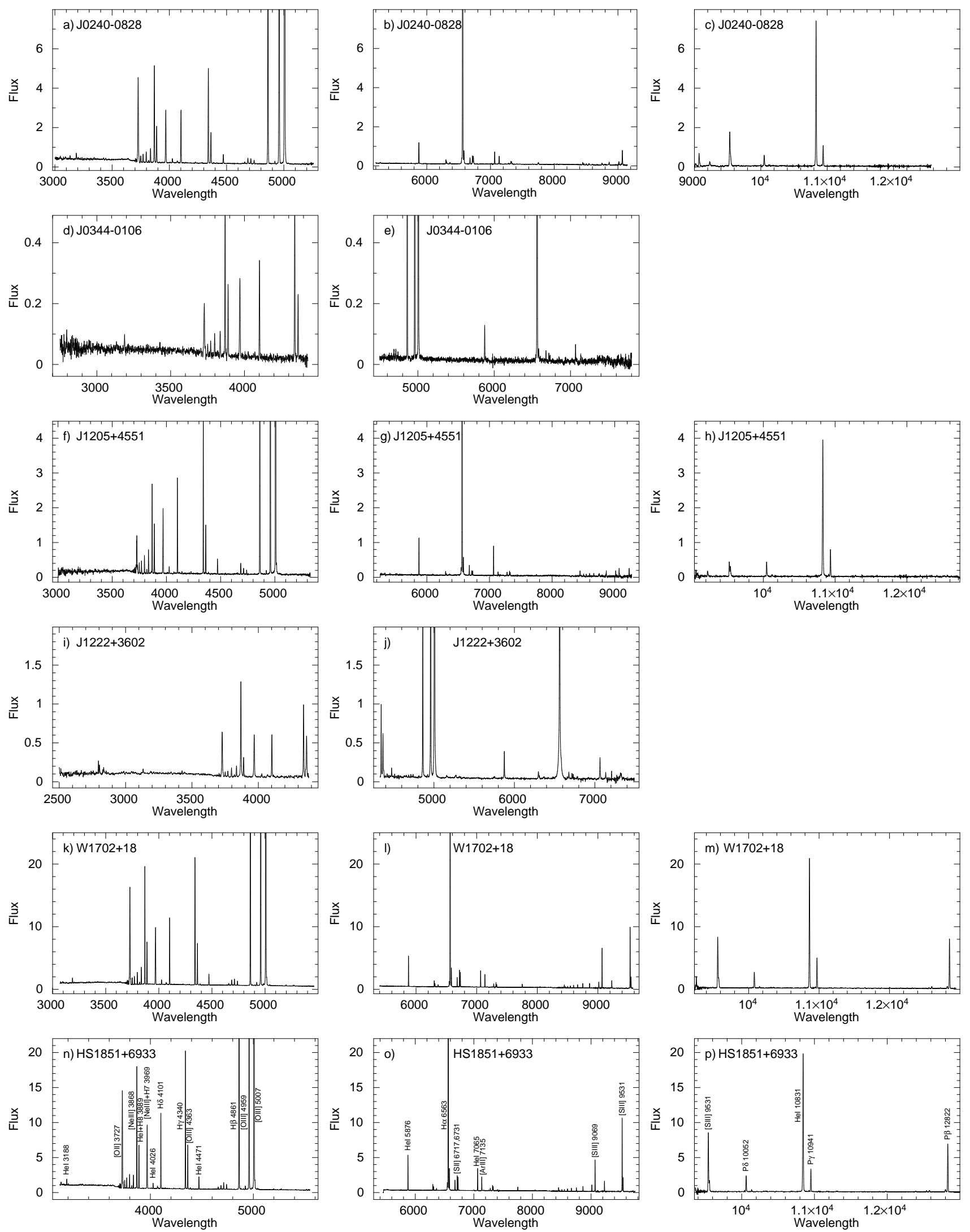

Figure A1. Rest-frame LBT spectra of selected galaxies in the blue (left) red (centre) and infrared (right) wavelength regions. Emission lines are labelled in the spectra of HS 1851+6933 in panels $\mathbf{n})-\mathbf{p}$ ). 
Table A1. Extinction-corrected emission-line fluxes ${ }^{\mathrm{a}}$

\begin{tabular}{|c|c|c|c|c|c|c|}
\hline Line & J0240-0828 & J0344-0106 & $\mathrm{J} 1205+4551$ & $\mathrm{~J} 1222+3602^{\mathrm{b}}$ & W $1702+18$ & HS $1851+6933$ \\
\hline $3096.00[\mathrm{Fe}$ IV] & $\ldots$ & $\ldots$ & $\ldots$ & $\ldots$ & $0.81 \pm 0.30$ & $\ldots$ \\
\hline 3132.79 O III & $\ldots$ & $\ldots$ & $\ldots$ & $2.70 \pm 0.25$ & $\ldots$ & $1.91 \pm 0.25$ \\
\hline 3187.74 He I & $4.09 \pm 0.30$ & $4.61 \pm 0.46$ & $1.21 \pm 0.17$ & $1.00 \pm 0.17$ & $2.81 \pm 0.22$ & $2.99 \pm 0.18$ \\
\hline 3203.10 He II & $\ldots$ & $\ldots$ & $1.21 \pm 0.18$ & $\ldots$ & $0.54 \pm 0.15$ & $0.61 \pm 0.12$ \\
\hline $3322.54[\mathrm{Fe} \mathrm{II}]$ & $\ldots$ & $\ldots$ & $\ldots$ & $\ldots$ & $0.33 \pm 0.12$ & $0.80 \pm 0.14$ \\
\hline 3340.74 O III & $0.97 \pm 0.12$ & $\ldots$ & $0.63 \pm 0.22$ & $\ldots$ & $0.44 \pm 0.09$ & $0.81 \pm 0.12$ \\
\hline $3370.00 \mathrm{~S}$ III & $\ldots$ & $\ldots$ & $\ldots$ & $\ldots$ & $\ldots$ & $0.27 \pm 0.05$ \\
\hline 3405.74 O III & $\ldots$ & & $0.32 \pm 0.13$ & $\ldots$ & $\ldots$ & $0.23 \pm 0.07$ \\
\hline $3426.85[\mathrm{Ne} \mathrm{v}]$ & $1.11 \pm 0.13$ & $1.62 \pm 0.32$ & $0.72 \pm 0.23$ & $1.31 \pm 0.17$ & $0.44 \pm 0.12$ & $0.22 \pm 0.06$ \\
\hline 3444.07 O III & $0.74 \pm 0.11$ & $\ldots$ & $\ldots$ & $1.02 \pm 0.18$ & $0.19 \pm 0.07$ & $0.31 \pm 0.08$ \\
\hline $3465.94 \mathrm{He} \mathrm{I}$ & $\ldots$ & $\ldots$ & $\ldots$ & $\ldots$ & $\ldots$ & $0.27 \pm 0.08$ \\
\hline $3487.27 \mathrm{He} \mathrm{I}$ & $\ldots$ & $\ldots$ & $\ldots$ & $\ldots$ & $\ldots$ & $0.17 \pm 0.06$ \\
\hline $3498.66 \mathrm{He} \mathrm{I}$ & $\ldots$ & $\ldots$ & $\ldots$ & $\ldots$ & $\ldots$ & $0.25 \pm 0.08$ \\
\hline $3530.09 \mathrm{He} \mathrm{I}$ & $\ldots$ & $\ldots$ & $0.55 \pm 0.20$ & $\ldots$ & $0.42 \pm 0.07$ & $0.25 \pm 0.01$ \\
\hline $3554.39 \mathrm{He} \mathrm{I}$ & $\ldots$ & $\ldots$ & $\ldots$ & $\ldots$ & $0.26 \pm 0.06$ & $0.27 \pm 0.06$ \\
\hline $3587.28 \mathrm{He} \mathrm{I}$ & $0.61 \pm 0.09$ & $\ldots$ & $\ldots$ & $\ldots$ & $0.15 \pm 0.03$ & $0.37 \pm 0.05$ \\
\hline $3612.48 \mathrm{He} \mathrm{I}$ & $\ldots$ & $\ldots$ & $\ldots$ & $\ldots$ & $0.27 \pm 0.05$ & $0.38 \pm 0.05$ \\
\hline $3634.25 \mathrm{He} \mathrm{I}$ & $\ldots$ & $\ldots$ & $0.41 \pm 0.11$ & $\ldots$ & $0.41 \pm 0.04$ & $0.36 \pm 0.04$ \\
\hline 3676.37 H22 & $\ldots$ & $\ldots$ & $\ldots$ & $\ldots$ & $0.26 \pm 0.03$ & $\ldots$ \\
\hline 3679.31 H21 & $\ldots$ & $\ldots$ & $\ldots$ & $\ldots$ & $0.26 \pm 0.03$ & $\ldots$ \\
\hline $3682.81 \mathrm{H} 20$ & $\ldots$ & $\ldots$ & $\ldots$ & $\ldots$ & $0.29 \pm 0.03$ & $\ldots$ \\
\hline 3686.83 H19 & $\ldots$ & $\ldots$ & $0.57 \pm 0.07$ & $\ldots$ & $0.44 \pm 0.03$ & $\ldots$ \\
\hline $3691.55 \mathrm{H} 18$ & $\ldots$ & $\ldots$ & $0.50 \pm 0.07$ & $\ldots$ & $0.65 \pm 0.04$ & $0.88 \pm 0.05$ \\
\hline 3697.15 H17 & $\ldots$ & $\ldots$ & $0.82 \pm 0.07$ & $\ldots$ & $0.98 \pm 0.06$ & $1.01 \pm 0.05$ \\
\hline $3703.30 \mathrm{H} 16$ & $2.04 \pm 0.09$ & $\ldots$ & $1.32 \pm 0.08$ & $\ldots$ & $1.88 \pm 0.08$ & $1.89 \pm 0.07$ \\
\hline 3711.97 H15 & $2.60 \pm 0.16$ & $\ldots$ & $2.85 \pm 0.21$ & $\ldots$ & $3.33 \pm 0.19$ & $2.30 \pm 0.11$ \\
\hline 3721.94 H14 & $\ldots$ & $\ldots$ & $1.49 \pm 0.08$ & $\ldots$ & $1.41 \pm 0.06$ & $1.18 \pm 0.05$ \\
\hline $3727.00[\mathrm{O}$ II] & $65.67 \pm 2.08$ & $20.17 \pm 0.74$ & $18.26 \pm 0.59$ & $36.42 \pm 1.21$ & $63.04 \pm 2.00$ & $59.79 \pm 1.79$ \\
\hline $3734.37 \mathrm{H} 13$ & $\ldots$ & $\ldots$ & $2.16 \pm 0.10$ & $2.04 \pm 0.18$ & $1.30 \pm 0.05$ & $1.92 \pm 0.07$ \\
\hline $3750.15 \mathrm{H} 12$ & $4.20 \pm 0.18$ & $4.71 \pm 0.44$ & $4.42 \pm 0.19$ & $4.83 \pm 0.42$ & $4.49 \pm 0.19$ & $3.78 \pm 0.14$ \\
\hline $3770.63 \mathrm{H} 11$ & $5.10 \pm 0.21$ & $4.97 \pm 0.45$ & $4.78 \pm 0.20$ & $5.59 \pm 0.40$ & $5.01 \pm 0.20$ & $4.83 \pm 0.17$ \\
\hline $3797.90 \mathrm{H} 10$ & $6.43 \pm 0.24$ & $6.58 \pm 0.46$ & $6.19 \pm 0.23$ & $6.85 \pm 0.39$ & $6.86 \pm 0.24$ & $5.97 \pm 0.20$ \\
\hline $3819.64 \mathrm{He} \mathrm{I}$ & $1.40 \pm 0.08$ & & $1.19 \pm 0.07$ & $1.71 \pm 0.18$ & $0.93 \pm 0.05$ & $1.01 \pm 0.05$ \\
\hline 3835.39 H9 & $8.31 \pm 0.29$ & $8.77 \pm 0.50$ & $8.15 \pm 0.28$ & $8.47 \pm 0.41$ & $8.72 \pm 0.29$ & $6.02 \pm 0.20$ \\
\hline 3868.76 [Ne III] & $53.79 \pm 1.67$ & $41.08 \pm 1.38$ & $24.25 \pm 0.76$ & $61.92 \pm 2.00$ & $49.50 \pm 1.54$ & $47.64 \pm 1.48$ \\
\hline $3889.00 \mathrm{He} \mathrm{I}+\mathrm{H} 8$ & $20.59 \pm 0.65$ & $19.27 \pm 0.74$ & $15.13 \pm 0.49$ & $13.61 \pm 0.54$ & $20.16 \pm 0.64$ & $17.00 \pm 0.53$ \\
\hline $3926.50 \mathrm{He} \mathrm{I}$ & $\ldots$ & $\ldots$ & $\ldots$ & $\ldots$ & $\ldots$ & $0.22 \pm 0.03$ \\
\hline $3968.00[\mathrm{Ne} \quad \mathrm{III}]+\mathrm{H} 7$ & $35.16 \pm 1.09$ & $28.44 \pm 1.00$ & $24.56 \pm 0.77$ & $37.65 \pm 1.24$ & $33.61 \pm 1.04$ & $34.06 \pm 1.04$ \\
\hline 4009.26 He I & $\ldots$ & & $0.42 \pm 0.05$ & $\ldots$ & $0.17 \pm 0.03$ & $0.29 \pm 0.03$ \\
\hline 4026.19 He I & $2.30 \pm 0.10$ & $1.87 \pm 0.23$ & $1.97 \pm 0.08$ & $2.46 \pm 0.20$ & $1.81 \pm 0.07$ & $1.92 \pm 0.07$ \\
\hline $4068.60[\mathrm{~S} \mathrm{II}]$ & $1.30 \pm 0.07$ & $\ldots$ & $0.65 \pm 0.06$ & $2.42 \pm 0.27$ & $1.00 \pm 0.06$ & $1.01 \pm 0.04$ \\
\hline 4076.35 [S II] & $\ldots$ & $\ldots$ & $0.30 \pm 0.06$ & $\ldots$ & $0.28 \pm 0.04$ & $0.32 \pm 0.02$ \\
\hline $4101.74 \mathrm{H} \delta$ & $28.49 \pm 0.87$ & $25.47 \pm 0.89$ & $27.50 \pm 0.84$ & $26.28 \pm 0.89$ & $28.34 \pm 0.86$ & $27.78 \pm 0.84$ \\
\hline 4120.89 He I & $\ldots$ & $\ldots$ & $0.44 \pm 0.05$ & $\ldots$ & $0.28 \pm 0.03$ & $0.23 \pm 0.02$ \\
\hline $4143.81 \mathrm{He} \mathrm{I}$ & $0.39 \pm 0.04$ & $\ldots$ & $0.57 \pm 0.05$ & $\ldots$ & $0.29 \pm 0.03$ & $0.36 \pm 0.03$ \\
\hline $4227.20[\mathrm{Fe} \mathrm{v}]$ & $0.72 \pm 0.06$ & $0.73 \pm 0.19$ & $0.73 \pm 0.05$ & $\ldots$ & $0.39 \pm 0.04$ & $0.32 \pm 0.03$ \\
\hline $4243.90[\mathrm{Fe} \mathrm{II}]$ & $\ldots$ & $\ldots$ & $\ldots$ & $\ldots$ & $\ldots$ & $0.09 \pm 0.02$ \\
\hline $4287.33[\mathrm{Fe}$ II] & $\ldots$ & $\ldots$ & $0.49 \pm 0.05$ & $\ldots$ & $0.21 \pm 0.03$ & $0.19 \pm 0.02$ \\
\hline $4340.47 \mathrm{H} \gamma$ & $49.14 \pm 1.44$ & $45.93 \pm 1.46$ & $46.99 \pm 1.44$ & $48.25 \pm 1.49$ & $48.64 \pm 1.43$ & $48.65 \pm 1.42$ \\
\hline $4363.21[\mathrm{O} \mathrm{III}]$ & $15.41 \pm 0.45$ & $16.36 \pm 0.63$ & $12.87 \pm 0.38$ & $29.52 \pm 0.91$ & $15.41 \pm 0.45$ & $15.03 \pm 0.44$ \\
\hline $4387.93 \mathrm{He} \mathrm{I}$ & $0.59 \pm 0.05$ & $\ldots$ & $0.49 \pm 0.05$ & $1.16 \pm 0.13$ & $0.43 \pm 0.03$ & $0.51 \pm 0.03$ \\
\hline $4415.00[\mathrm{Fe} \mathrm{II}]$ & $0.40 \pm 0.05$ & $\ldots$ & $\ldots$ & $2.59 \pm 0.16$ & $0.15 \pm 0.03$ & $0.26 \pm 0.02$ \\
\hline $4437.55 \mathrm{He} \mathrm{I}$ & $\ldots$ & $\ldots$ & $\ldots$ & $1.53 \pm 0.13$ & $0.08 \pm 0.02$ & $0.15 \pm 0.02$ \\
\hline $4452.02[\mathrm{Fe} \mathrm{II}]$ & $\ldots$ & $\ldots$ & $\ldots$ & $1.28 \pm 0.11$ & $\ldots$ & $0.08 \pm 0.01$ \\
\hline $4471.48 \mathrm{He} \mathrm{I}$ & $4.62 \pm 0.15$ & $\ldots$ & $4.02 \pm 0.13$ & $4.68 \pm 0.21$ & $3.97 \pm 0.12$ & $4.18 \pm 0.12$ \\
\hline $4514.89 \mathrm{~N}$ III & $\ldots$ & $\ldots$ & $\ldots$ & $\ldots$ & $\ldots$ & $0.10 \pm 0.03$ \\
\hline $4562.50[\mathrm{Mg} \mathrm{I}]$ & $\ldots$ & $\ldots$ & $\ldots$ & $1.62 \pm 0.11$ & $0.15 \pm 0.02$ & $0.13 \pm 0.02$ \\
\hline $4571.10 \mathrm{Mg} \mathrm{I}]$ & $\ldots$ & $\ldots$ & $\ldots$ & $\ldots$ & $0.09 \pm 0.02$ & $0.08 \pm 0.01$ \\
\hline $4634.14 \mathrm{~N}$ III & $\ldots$ & $\ldots$ & $\ldots$ & $\ldots$ & $\ldots$ & $0.06 \pm 0.02$ \\
\hline $4640.00 \mathrm{~N}$ III & $\ldots$ & $\ldots$ & $\ldots$ & $0.66 \pm 0.09$ & $0.07 \pm 0.02$ & $0.14 \pm 0.02$ \\
\hline $4640.00 \mathrm{WR}$ & $0.49 \pm 0.15$ & $\ldots$ & $0.87 \pm 0.18$ & $2.50 \pm 0.31$ & $0.20 \pm 0.05$ & $0.20 \pm 0.04$ \\
\hline $4658.10[\mathrm{Fe} \quad \mathrm{III}]$ & $0.84 \pm 0.06$ & $\ldots$ & $0.58 \pm 0.05$ & $0.72 \pm 0.08$ & $0.65 \pm 0.04$ & $0.69 \pm 0.03$ \\
\hline $4685.94 \mathrm{He}$ II & $2.96 \pm 0.11$ & $3.41 \pm 0.16$ & $3.24 \pm 0.11$ & $1.13 \pm 0.11$ & $1.96 \pm 0.07$ & $1.39 \pm 0.05$ \\
\hline
\end{tabular}


Table A1 - continued Extinction-corrected emission-line fluxes ${ }^{\mathrm{a}}$

\begin{tabular}{|c|c|c|c|c|c|c|}
\hline Line & J0240-0828 & J0344-0106 & $\mathrm{J} 1205+4551$ & $\mathrm{~J} 1222+3602^{\mathrm{b}}$ & $\mathrm{W} 1702+18$ & HS $1851+6933$ \\
\hline $4701.56[\mathrm{Fe} \mathrm{III}]$ & & & & & $0.18 \pm 0.03$ & $0.20 \pm 0.02$ \\
\hline $4712.00[\mathrm{Ar} \mathrm{IV}]+\mathrm{He} \mathrm{I}_{\mathrm{I}}$ & $2.32 \pm 0.09$ & $3.16 \pm 0.15$ & $2.15 \pm 0.08$ & $1.61 \pm 0.12$ & $2.29 \pm 0.07$ & $2.37 \pm 0.07$ \\
\hline $4740.20[\mathrm{Ar} \mathrm{IV}]$ & $1.70 \pm 0.07$ & $2.41 \pm 0.15$ & $1.20 \pm 0.06$ & $1.27 \pm 0.11$ & $1.37 \pm 0.05$ & $1.56 \pm 0.05$ \\
\hline $4754.72[\mathrm{Fe} \mathrm{III}]$ & $\ldots$ & $\ldots$ & $0.31 \pm 0.03$ & $\ldots$ & $0.13 \pm 0.02$ & $0.19 \pm 0.02$ \\
\hline $4769.60[\mathrm{Fe} \quad \mathrm{III}]$ & $\ldots$ & $\ldots$ & $\ldots$ & $\ldots$ & $\ldots$ & $0.07 \pm 0.01$ \\
\hline $4788.09 \mathrm{~N}$ II & $\ldots$ & $\ldots$ & $\ldots$ & $\ldots$ & $\ldots$ & $0.12 \pm 0.02$ \\
\hline $4814.47[\mathrm{Fe} \mathrm{II}]$ & $\ldots$ & $\ldots$ & $0.26 \pm 0.04$ & $\ldots$ & $0.09 \pm 0.02$ & $0.14 \pm 0.02$ \\
\hline $4861.33 \mathrm{H} \beta$ & $100.00 \pm 2.85$ & $100.00 \pm 2.91$ & $100.00 \pm 2.86$ & $100.00 \pm 2.92$ & $100.00 \pm 2.86$ & $100.00 \pm 2.84$ \\
\hline $4881.01[\mathrm{Fe} \mathrm{III}]$ & $\ldots$ & $\ldots$ & $\ldots$ & $\ldots$ & $0.28 \pm 0.03$ & $0.28 \pm 0.02$ \\
\hline 4901.11 [Fe IV] & $\ldots$ & $\ldots$ & $\ldots$ & $\ldots$ & $0.11 \pm 0.02$ & $0.11 \pm 0.01$ \\
\hline $4905.00[\mathrm{Fe} \mathrm{II}]$ & $\ldots$ & $\ldots$ & $\ldots$ & $\ldots$ & $0.13 \pm 0.02$ & $0.21 \pm 0.02$ \\
\hline $4921.93 \mathrm{He} \mathrm{I}$ & $1.06 \pm 0.06$ & $\ldots$ & $1.03 \pm 0.05$ & $1.94 \pm 0.14$ & $1.01 \pm 0.04$ & $1.04 \pm 0.04$ \\
\hline $4930.50[\mathrm{Fe} \mathrm{III}]$ & $\ldots$ & $\ldots$ & $\ldots$ & $\ldots$ & $0.09 \pm 0.01$ & $0.18 \pm 0.02$ \\
\hline $4958.92[\mathrm{O} \quad \mathrm{III}]$ & $239.06 \pm 6.81$ & $199.09 \pm 5.76$ & $139.15 \pm 3.98$ & $281.07 \pm 8.16$ & $205.07 \pm 5.87$ & $218.41 \pm 6.21$ \\
\hline $4986.00[\mathrm{Fe} \mathrm{III}]$ & $\ldots$ & $\ldots$ & & $\ldots$ & $0.62 \pm 0.03$ & $0.50 \pm 0.02$ \\
\hline $5006.80[\mathrm{O} \mathrm{III}]$ & $713.61 \pm 20.3$ & $608.79 \pm 17.6$ & $414.63 \pm 11.9$ & $832.21 \pm 24.2$ & $609.85 \pm 17.0$ & $654.13 \pm 18.6$ \\
\hline $5015.68 \mathrm{He} \mathrm{I}$ & $\ldots$ & $\ldots$ & $1.98 \pm 0.07$ & $\ldots$ & $2.03 \pm 0.07$ & $1.91 \pm 0.06$ \\
\hline $5041.03 \mathrm{Si} \mathrm{II}$ & $\ldots$ & $\ldots$ & $0.39 \pm 0.04$ & $\ldots$ & $0.33 \pm 0.03$ & $0.37 \pm 0.02$ \\
\hline $5047.80 \mathrm{He}$ I & $\ldots$ & $\ldots$ & $0.43 \pm 0.06$ & $\ldots$ & $0.18 \pm 0.02$ & $0.23 \pm 0.02$ \\
\hline $5056.05 \mathrm{Si}$ II & $\ldots$ & $\ldots$ & $0.36 \pm 0.04$ & $\ldots$ & $0.14 \pm 0.02$ & $0.20 \pm 0.02$ \\
\hline 5160.00 [Fe II] & $\ldots$ & $\ldots$ & $\ldots$ & $2.15 \pm 0.12$ & $0.11 \pm 0.02$ & $0.14 \pm 0.02$ \\
\hline 5191.82 [Ar III] & $\ldots$ & $\ldots$ & $\ldots$ & $\ldots$ & $0.06 \pm 0.02$ & $\ldots$ \\
\hline $5199.00[\mathrm{~N} \mathrm{I}]$ & $\ldots$ & $\ldots$ & $\ldots$ & $\ldots$ & $0.33 \pm 0.03$ & $0.37 \pm 0.02$ \\
\hline 5233.76 [Fe IV] & $\ldots$ & $\ldots$ & $\ldots$ & $\ldots$ & $0.08 \pm 0.02$ & $0.13 \pm 0.02$ \\
\hline $5261.62[\mathrm{Fe} \mathrm{II}]$ & $0.14 \pm 0.03$ & $\ldots$ & $\ldots$ & $0.99 \pm 0.08$ & $0.13 \pm 0.02$ & $0.18 \pm 0.02$ \\
\hline 5270.63 [Fe III] & $\ldots$ & $\ldots$ & $\ldots$ & $\ldots$ & $0.35 \pm 0.03$ & $0.39 \pm 0.02$ \\
\hline 5323.27 [Cl IV] & $\ldots$ & $\ldots$ & $\ldots$ & $\ldots$ & $\ldots$ & $0.07 \pm 0.01$ \\
\hline 5411.52 He II & $0.29 \pm 0.03$ & $\ldots$ & $0.32 \pm 0.03$ & $\ldots$ & $0.11 \pm 0.03$ & $0.11 \pm 0.02$ \\
\hline $5517.71[\mathrm{Cl} \mathrm{III}]$ & $0.30 \pm 0.02$ & $\ldots$ & $\ldots$ & $\ldots$ & $0.18 \pm 0.02$ & $0.30 \pm 0.02$ \\
\hline 5537.88 [Cl III] & $0.10 \pm 0.02$ & $\ldots$ & & $\ldots$ & $0.08 \pm 0.01$ & $0.20 \pm 0.02$ \\
\hline $5754.64[\mathrm{~N} \mathrm{II}]$ & $0.30 \pm 0.02$ & $\ldots$ & $0.32 \pm 0.03$ & $0.98 \pm 0.10$ & $0.11 \pm 0.01$ & $0.14 \pm 0.01$ \\
\hline $5875.60 \mathrm{He} \mathrm{I}$ & $11.87 \pm 0.36$ & $10.90 \pm 0.37$ & $11.77 \pm 0.35$ & $17.47 \pm 0.58$ & $10.46 \pm 0.31$ & $11.60 \pm 0.34$ \\
\hline $5957.55 \mathrm{Si}$ II & $0.20 \pm 0.02$ & $\ldots$ & $\ldots$ & $\ldots$ & $0.08 \pm 0.01$ & $0.10 \pm 0.01$ \\
\hline $5978.91 \mathrm{Si}$ II & $0.21 \pm 0.02$ & $\ldots$ & $\ldots$ & $\ldots$ & $0.11 \pm 0.01$ & $0.12 \pm 0.01$ \\
\hline 6046.00 O I & $\ldots$ & $\ldots$ & $\ldots$ & $\ldots$ & $0.03 \pm 0.01$ & $0.05 \pm 0.01$ \\
\hline 6087.00 [Fe VII] & $\ldots$ & $\ldots$ & $0.11 \pm 0.02$ & $\ldots$ & $\ldots$ & $0.03 \pm 0.01$ \\
\hline $6102.00 \mathrm{He}$ II & $0.07 \pm 0.01$ & $\ldots$ & $\ldots$ & $\ldots$ & $0.09 \pm 0.01$ & $0.08 \pm 0.01$ \\
\hline $6300.30[\mathrm{O}$ I $]$ & $2.16 \pm 0.07$ & $\ldots$ & $1.21 \pm 0.04$ & $5.58 \pm 0.23$ & $1.90 \pm 0.06$ & $1.81 \pm 0.06$ \\
\hline 6312.10 [S III] & $1.01 \pm 0.04$ & $\ldots$ & $0.50 \pm 0.03$ & $2.26 \pm 0.12$ & $1.07 \pm 0.04$ & $1.13 \pm 0.04$ \\
\hline $6347.08 \mathrm{Si}$ II & $\ldots$ & $\ldots$ & $0.13 \pm 0.02$ & $\ldots$ & $0.08 \pm 0.01$ & $0.13 \pm 0.01$ \\
\hline $6363.80[\mathrm{O}$ I] & $0.75 \pm 0.04$ & $\ldots$ & $0.36 \pm 0.03$ & $1.48 \pm 0.12$ & $0.63 \pm 0.02$ & $0.61 \pm 0.02$ \\
\hline 6371.36 Si II & $\ldots$ & $\ldots$ & $\ldots$ & $\ldots$ & $0.11 \pm 0.01$ & $\ldots$ \\
\hline $6548.10[\mathrm{~N} \mathrm{II}]$ & $\ldots$ & $1.24 \pm 0.09$ & $1.65 \pm 0.06$ & $\ldots$ & $1.37 \pm 0.04$ & $1.95 \pm 0.06$ \\
\hline $6562.80 \mathrm{H} \alpha$ & $282.31 \pm 8.70$ & $277.88 \pm 8.70$ & $278.70 \pm 8.62$ & $360.21 \pm 11.3$ & $282.48 \pm 8.75$ & $282.07 \pm 8.69$ \\
\hline $6583.40[\mathrm{~N} \mathrm{II}]$ & $8.09 \pm 0.25$ & $3.43 \pm 0.15$ & $4.61 \pm 0.15$ & $3.14 \pm 0.20$ & $5.37 \pm 0.17$ & $6.15 \pm 0.19$ \\
\hline $6678.10 \mathrm{He} \mathrm{I}$ & $3.46 \pm 0.11$ & $2.77 \pm 0.15$ & $2.91 \pm 0.10$ & $4.15 \pm 0.19$ & $2.88 \pm 0.09$ & $3.07 \pm 0.10$ \\
\hline 6716.40 [S II] & $4.74 \pm 0.15$ & $2.72 \pm 0.16$ & $1.34 \pm 0.05$ & $2.51 \pm 0.15$ & $5.06 \pm 0.16$ & $4.35 \pm 0.14$ \\
\hline 6730.80 [S II] & $4.44 \pm 0.14$ & $1.95 \pm 0.14$ & $1.31 \pm 0.05$ & $2.52 \pm 0.14$ & $4.36 \pm 0.14$ & $4.01 \pm 0.13$ \\
\hline $6739.80[\mathrm{Fe}$ IV] & $\ldots$ & $\ldots$ & $\ldots$ & $\ldots$ & $0.09 \pm 0.01$ & $0.16 \pm 0.01$ \\
\hline $6933.91 \mathrm{He} \mathrm{I}$ & $\ldots$ & $\ldots$ & $\ldots$ & $\ldots$ & $0.06 \pm 0.01$ & $\ldots$ \\
\hline $7002.00 \mathrm{O}$ I & $\ldots$ & $\ldots$ & $\ldots$ & $\ldots$ & $\ldots$ & $0.16 \pm 0.01$ \\
\hline $7065.30 \mathrm{He} \mathrm{I}$ & $5.68 \pm 0.19$ & $4.78 \pm 0.21$ & $7.87 \pm 0.25$ & $15.89 \pm 0.58$ & $4.66 \pm 0.15$ & $6.36 \pm 0.20$ \\
\hline 7135.80 [Ar III] & $3.94 \pm 0.13$ & $2.16 \pm 0.14$ & $1.03 \pm 0.05$ & $4.05 \pm 0.24$ & $3.62 \pm 0.12$ & $3.85 \pm 0.12$ \\
\hline 7155.08 [Fe II] & $\ldots$ & $\ldots$ & $\ldots$ & $\ldots$ & $0.03 \pm 0.01$ & $0.09 \pm 0.01$ \\
\hline $7170.62[\mathrm{Ar}$ IV $]$ & $\ldots$ & $\ldots$ & $\ldots$ & $\ldots$ & $0.10 \pm 0.01$ & $0.13 \pm 0.01$ \\
\hline $7237.00[\mathrm{Ar}$ IV] & $\ldots$ & $\ldots$ & $\ldots$ & $\ldots$ & $0.08 \pm 0.01$ & $0.06 \pm 0.01$ \\
\hline $7254.00 \mathrm{O}_{\mathrm{I}}$ & $\ldots$ & $\ldots$ & $\ldots$ & $\ldots$ & $0.11 \pm 0.01$ & $0.14 \pm 0.01$ \\
\hline 7262.76 [Ar IV] & $\ldots$ & $\ldots$ & $\ldots$ & $\ldots$ & $0.06 \pm 0.01$ & $0.08 \pm 0.01$ \\
\hline $7281.20 \mathrm{He}$ I & $0.72 \pm 0.03$ & $\ldots$ & $0.89 \pm 0.03$ & $\ldots$ & $1.00 \pm 0.06$ & $0.68 \pm 0.02$ \\
\hline $7298.00 \mathrm{He} \mathrm{I}$ & $\ldots$ & $\ldots$ & $\ldots$ & $\ldots$ & $\ldots$ & $0.04 \pm 0.01$ \\
\hline $7319.90[\mathrm{O} \quad \mathrm{II}]$ & $1.57 \pm 0.06$ & $\ldots$ & $1.13 \pm 0.04$ & $5.50 \pm 0.36$ & $1.19 \pm 0.04$ & $1.51 \pm 0.05$ \\
\hline $7330.20[\mathrm{O} \quad \mathrm{II}]$ & $1.20 \pm 0.05$ & $\ldots$ & $0.87 \pm 0.04$ & $3.63 \pm 0.34$ & $0.75 \pm 0.03$ & $1.17 \pm 0.04$ \\
\hline $7377.83[\mathrm{Ni} I \mathrm{II}]$ & $\ldots$ & $\ldots$ & $\ldots$ & $\ldots$ & $0.11 \pm 0.01$ & $0.12 \pm 0.01$ \\
\hline
\end{tabular}


Table A1 - continued Extinction-corrected emission-line fluxes ${ }^{\mathrm{a}}$

\begin{tabular}{|c|c|c|c|c|c|c|}
\hline Line & J0240-0828 & J0344-0106 & $\mathrm{J} 1205+4551$ & $\mathrm{~J} 1222+3602^{\mathrm{b}}$ & W $1702+18$ & HS $1851+6933$ \\
\hline $7468.31 \mathrm{~N} \mathrm{I}$ & $\ldots$ & $\ldots$ & $\cdots$ & $\ldots$ & $0.06 \pm 0.01$ & $\cdots$ \\
\hline 7499.85 He I & $\ldots$ & $\ldots$ & $\ldots$ & $\ldots$ & $0.06 \pm 0.01$ & $\ldots$ \\
\hline 7751.12 [Ar III] & $1.02 \pm 0.04$ & $\ldots$ & $0.33 \pm 0.02$ & $\ldots$ & $0.84 \pm 0.03$ & $1.00 \pm 0.04$ \\
\hline 7816.80 He II & $\ldots$ & $\ldots$ & $\ldots$ & $\ldots$ & $0.06 \pm 0.01$ & $0.06 \pm 0.01$ \\
\hline 8045.63 [Cl IV] & $0.14 \pm 0.02$ & $\ldots$ & $\ldots$ & $\ldots$ & $0.19 \pm 0.01$ & $0.27 \pm 0.01$ \\
\hline 8298.83 P28 & $\ldots$ & $\ldots$ & $\ldots$ & $\ldots$ & $\ldots$ & $0.08 \pm 0.01$ \\
\hline 8306.22 P27 & $\ldots$ & $\ldots$ & $\ldots$ & $\ldots$ & $\ldots$ & $0.09 \pm 0.01$ \\
\hline 8314.26 P26 & $\ldots$ & $\ldots$ & $\ldots$ & $\ldots$ & $0.05 \pm 0.01$ & $0.09 \pm 0.01$ \\
\hline 8323.43 P25 & $\ldots$ & $\ldots$ & $\ldots$ & $\ldots$ & $0.06 \pm 0.01$ & $0.09 \pm 0.01$ \\
\hline 8333.78 P24 & $\ldots$ & $\ldots$ & $0.16 \pm 0.02$ & $\ldots$ & $0.11 \pm 0.01$ & $0.14 \pm 0.01$ \\
\hline 8345.44 P23 & $\ldots$ & $\ldots$ & $\ldots$ & $\ldots$ & $0.13 \pm 0.01$ & $0.17 \pm 0.01$ \\
\hline 8359.01 P22 & $0.16 \pm 0.02$ & $\ldots$ & $\ldots$ & $\ldots$ & $0.24 \pm 0.01$ & $0.29 \pm 0.01$ \\
\hline 8374.48 P21 & $0.18 \pm 0.02$ & $\ldots$ & $\ldots$ & $\ldots$ & $0.19 \pm 0.01$ & $0.19 \pm 0.01$ \\
\hline 8392.40 P20 & $0.17 \pm 0.02$ & $\ldots$ & $\ldots$ & $\ldots$ & $0.23 \pm 0.02$ & $0.23 \pm 0.01$ \\
\hline 8413.32 P19 & $0.26 \pm 0.02$ & $\ldots$ & $0.22 \pm 0.02$ & $\ldots$ & $0.28 \pm 0.02$ & $0.23 \pm 0.01$ \\
\hline 8437.96 P18 & $\ldots$ & $\ldots$ & $0.21 \pm 0.03$ & $\ldots$ & $0.33 \pm 0.02$ & $0.27 \pm 0.01$ \\
\hline 8446.34 O I & $0.96 \pm 0.04$ & $\ldots$ & $1.41 \pm 0.06$ & $\ldots$ & $0.71 \pm 0.03$ & $0.92 \pm 0.03$ \\
\hline $8467.26 \mathrm{P} 17$ & $0.45 \pm 0.03$ & $\ldots$ & $0.33 \pm 0.03$ & $\ldots$ & $0.31 \pm 0.02$ & $0.31 \pm 0.01$ \\
\hline 8502.49 P16 & $0.52 \pm 0.03$ & $\ldots$ & $0.63 \pm 0.04$ & $\ldots$ & $0.39 \pm 0.02$ & $0.45 \pm 0.02$ \\
\hline 8545.38 P15 & $0.65 \pm 0.04$ & $\ldots$ & $0.65 \pm 0.04$ & $\ldots$ & $0.53 \pm 0.02$ & $0.48 \pm 0.02$ \\
\hline 8598.39 P14 & $0.35 \pm 0.03$ & $\ldots$ & $0.68 \pm 0.04$ & $\ldots$ & $0.64 \pm 0.03$ & $0.66 \pm 0.03$ \\
\hline 8665.02 P13 & $\ldots$ & $\ldots$ & $0.90 \pm 0.05$ & $\ldots$ & $0.76 \pm 0.03$ & $0.76 \pm 0.03$ \\
\hline $8683.40 \mathrm{~N} \mathrm{I}$ & $\ldots$ & $\ldots$ & $\ldots$ & $\ldots$ & $0.12 \pm 0.01$ & $0.12 \pm 0.01$ \\
\hline 8750.47 P12 & $0.73 \pm 0.04$ & $\ldots$ & $\ldots$ & $\ldots$ & $1.05 \pm 0.04$ & $0.99 \pm 0.04$ \\
\hline 8862.79 P11 & $1.13 \pm 0.06$ & $\ldots$ & $1.20 \pm 0.07$ & $\ldots$ & $1.13 \pm 0.05$ & $1.27 \pm 0.05$ \\
\hline 8996.99 He I & $\ldots$ & $\ldots$ & $\ldots$ & $\ldots$ & $\ldots$ & $0.09 \pm 0.01$ \\
\hline 9014.91 P10 & $1.44 \pm 0.08$ & $\ldots$ & $1.25 \pm 0.06$ & $\ldots$ & $1.64 \pm 0.07$ & $1.68 \pm 0.06$ \\
\hline 9069.00 [S III $]$ & $6.70 \pm 0.26$ & $\ldots$ & $2.05 \pm 0.10$ & $\ldots$ & $8.02 \pm 0.38$ & $7.94 \pm 0.29$ \\
\hline 9229.02 P9 & $1.68 \pm 0.10$ & $\ldots$ & $2.06 \pm 0.10$ & $\ldots$ & $1.77 \pm 0.08$ & $2.48 \pm 0.10$ \\
\hline $9463.62 \mathrm{He} \mathrm{I}$ & $\ldots$ & $\ldots$ & $\ldots$ & $\ldots$ & $0.07 \pm 0.03$ & $0.18 \pm 0.02$ \\
\hline 9530.60 [S III] & $23.02 \pm 0.86$ & $\ldots$ & $4.60 \pm 0.18$ & $\ldots$ & $14.72 \pm 0.56$ & $18.42 \pm 0.69$ \\
\hline $9545.98 \mathrm{P} 8$ & $\ldots$ & $\ldots$ & $3.51 \pm 0.14$ & $\ldots$ & $2.74 \pm 0.12$ & $3.53 \pm 0.14$ \\
\hline 10030.00 He I & $\ldots$ & $\ldots$ & $\ldots$ & $\ldots$ & $0.31 \pm 0.01$ & $0.34 \pm 0.04$ \\
\hline $10052.15 \mathrm{P} \delta$ & $\ldots$ & $\ldots$ & $5.75 \pm 0.17$ & $\ldots$ & $6.25 \pm 0.17$ & $5.89 \pm 0.17$ \\
\hline $10290.00[\mathrm{~S} \mathrm{II}]$ & $\ldots$ & $\ldots$ & $\ldots$ & $\ldots$ & $0.33 \pm 0.03$ & $0.19 \pm 0.02$ \\
\hline $10320.00[\mathrm{~S}$ II] & $\ldots$ & $\ldots$ & $\ldots$ & $\ldots$ & $0.43 \pm 0.03$ & $\ldots$ \\
\hline 10831.00 He I & $84.21 \pm 2.49$ & $\ldots$ & $62.96 \pm 1.86$ & $\ldots$ & $50.59 \pm 1.34$ & $55.82 \pm 1.63$ \\
\hline 10910.00 He I & $\ldots$ & $\ldots$ & $\ldots$ & $\ldots$ & $\ldots$ & $0.38 \pm 0.04$ \\
\hline $10941.12 \mathrm{P} \gamma$ & $10.84 \pm 0.32$ & $\ldots$ & $9.66 \pm 0.29$ & $\ldots$ & $10.73 \pm 0.29$ & $7.94 \pm 0.24$ \\
\hline 11630.00 He II & $\ldots$ & $\ldots$ & $\ldots$ & $\ldots$ & $\ldots$ & $0.11 \pm 0.01$ \\
\hline $11970.00 \mathrm{He} \mathrm{I}$ & $\ldots$ & $\ldots$ & $\ldots$ & $\ldots$ & $0.31 \pm 0.02$ & $0.28 \pm 0.02$ \\
\hline $12530.00 \mathrm{He} \mathrm{I}$ & $\ldots$ & $\ldots$ & $\ldots$ & $\ldots$ & $0.30 \pm 0.03$ & $0.41 \pm 0.03$ \\
\hline $12570.00[\mathrm{Fe} \mathrm{II}]$ & $\ldots$ & $\ldots$ & $\ldots$ & $\ldots$ & $0.61 \pm 0.04$ & $0.59 \pm 0.03$ \\
\hline $12790.00 \mathrm{He}$ I & $\ldots$ & $\ldots$ & $\ldots$ & $\ldots$ & $0.83 \pm 0.04$ & $0.96 \pm 0.04$ \\
\hline $12821.62 \mathrm{P} \beta$ & $\ldots$ & $\ldots$ & $\ldots$ & $\ldots$ & $17.29 \pm 0.48$ & $16.66 \pm 0.51$ \\
\hline$C(\mathrm{H} \beta)^{\mathrm{c}}$ & $0.330 \pm 0.037$ & $0.150 \pm 0.037$ & $0.310 \pm 0.037$ & $0.105 \pm 0.037$ & $0.440 \pm 0.037$ & $0.410 \pm 0.037$ \\
\hline$F(\mathrm{H} \beta)^{\mathrm{d}}$ & $56.3 \pm 0.2$ & $5.86 \pm 0.2$ & $44.4 \pm 0.1$ & $10.5 \pm 0.1$ & $196.0 \pm 0.2$ & $183.3 \pm 0.2$ \\
\hline $\mathrm{EW}(\mathrm{H} \beta)^{\mathrm{e}}$ & $345.1 \pm 1.0$ & $311.8 \pm 1.2$ & $519.4 \pm 0.9$ & $190.4 \pm 0.6$ & $309.8 \pm 1.0$ & $412.6 \pm 1.0$ \\
\hline $\mathrm{EW}(\mathrm{abs})^{\mathrm{e}}$ & $1.2 \pm 0.1$ & $3.0 \pm 0.3$ & $3.6 \pm 0.2$ & $3.6 \pm 0.2$ & $3.0 \pm 0.1$ & $1.3 \pm 0.1$ \\
\hline
\end{tabular}

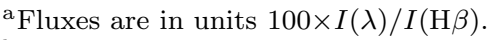

${ }^{\mathrm{b}}$ Corrected with extinction coefficient derived excluding the $\mathrm{H} \alpha$ emission line.

${ }^{\mathrm{c}}$ Extinction coefficient, derived from the observed hydrogen Balmer decrement.

${ }^{\mathrm{d}}$ Observed flux in units of $10^{-16} \mathrm{erg} \mathrm{s}^{-1} \mathrm{~cm}^{-2}$.

e Equivalent width in $\AA$. 
Table A2. Electron temperatures, electron number densities, ionic and elemental abundances

\begin{tabular}{|c|c|c|c|c|c|c|}
\hline Property & J0240-0828 & J0344-0106 & $\mathrm{J} 1205+4551$ & $\mathrm{~J} 1222+3602$ & W $1702+18$ & HS $1851+6933$ \\
\hline$T_{\mathrm{e}}(\mathrm{O}$ III $), \mathrm{K}$ & $16000 \pm 300$ & $17700 \pm 400$ & $19000 \pm 400$ & $20600 \pm 500$ & $17000 \pm 300$ & $16300 \pm 300$ \\
\hline$T_{\mathrm{e}}(\mathrm{O}$ II $), \mathrm{K}$ & $14500 \pm 200$ & $15200 \pm 300$ & $15500 \pm 300$ & $15600 \pm 300$ & $15000 \pm 300$ & $14600 \pm 300$ \\
\hline$T_{\mathrm{e}}(\mathrm{S}$ III $), \mathrm{K}$ & $14600 \pm 200$ & $16600 \pm 400$ & $17900 \pm 300$ & $18100 \pm 400$ & $15900 \pm 300$ & $15100 \pm 300$ \\
\hline$N_{\mathrm{e}}(\mathrm{S}$ II $), \mathrm{cm}^{-3}$ & $485 \pm 103$ & $28 \pm 78$ & $616 \pm 142$ & $676 \pm 227$ & $319 \pm 86$ & $449 \pm 98$ \\
\hline$N_{\mathrm{e}}(\mathrm{He} \mathrm{I}), \mathrm{cm}^{-3}$ & $674 \pm 150$ & $\ldots$ & $512 \pm 130$ & $\ldots$ & $295 \pm 70$ & $684 \pm 160$ \\
\hline $\mathrm{O}^{+} / \mathrm{H}^{+} \times 10^{5}$ & $0.697 \pm 0.039$ & $0.177 \pm 0.012$ & $0.160 \pm 0.010$ & $0.312 \pm 0.020$ & $0.597 \pm 0.034$ & $0.583 \pm 0.032$ \\
\hline $\mathrm{O}^{2+} / \mathrm{H}^{+} \times 10^{5}$ & $6.745 \pm 0.341$ & $4.519 \pm 0.278$ & $2.631 \pm 0.144$ & $4.496 \pm 0.262$ & $4.949 \pm 0.256$ & $5.909 \pm 0.299$ \\
\hline $\mathrm{O}^{3+} / \mathrm{H}^{+} \times 10^{6}$ & $1.965 \pm 0.132$ & $1.769 \pm 0.164$ & $0.943 \pm 0.066$ & $0.393 \pm 0.048$ & $1.161 \pm 0.077$ & $0.903 \pm 0.060$ \\
\hline $\mathrm{O} / \mathrm{H} \times 10^{5}$ & $7.639 \pm 0.344$ & $4.873 \pm 0.278$ & $2.886 \pm 0.145$ & $4.848 \pm 0.263$ & $5.662 \pm 0.259$ & $6.582 \pm 0.300$ \\
\hline $12+\log (\mathrm{O} / \mathrm{H})$ & $7.883 \pm 0.020$ & $7.688 \pm 0.028$ & $7.460 \pm 0.022$ & $7.686 \pm 0.024$ & $7.753 \pm 0.020$ & $7.818 \pm 0.020$ \\
\hline $\mathrm{N}^{+} / \mathrm{H}^{+} \times 10^{6}$ & $0.640 \pm 0.025$ & $0.247 \pm 0.013$ & $0.320 \pm 0.014$ & $0.215 \pm 0.013$ & $0.399 \pm 0.016$ & $0.477 \pm 0.018$ \\
\hline $\mathrm{ICF}(\mathrm{N})$ & 9.721 & 16.058 & 23.863 & 13.787 & 8.631 & 10.074 \\
\hline $\mathrm{N} / \mathrm{H} \times 10^{6}$ & $6.222 \pm 0.272$ & $5.893 \pm 0.351$ & $5.139 \pm 0.245$ & $2.960 \pm 0.208$ & $3.442 \pm 0.150$ & $4.807 \pm 0.208$ \\
\hline $\log (\mathrm{N} / \mathrm{O})$ & $-1.089 \pm 0.027$ & $-0.917 \pm 0.036$ & $-0.749 \pm 0.030$ & $-1.214 \pm 0.039$ & $-1.216 \pm 0.027$ & $-1.137 \pm 0.027$ \\
\hline $\mathrm{Ne}^{2+} / \mathrm{H}^{+} \times 10^{5}$ & $1.207 \pm 0.067$ & $0.704 \pm 0.045$ & $0.345 \pm 0.020$ & $0.734 \pm 0.043$ & $0.931 \pm 0.052$ & $1.015 \pm 0.056$ \\
\hline $\mathrm{ICF}(\mathrm{Ne})$ & 1.041 & 1.025 & 1.037 & 1.025 & 1.050 & 1.034 \\
\hline $\mathrm{Ne} / \mathrm{H} \times 10^{5}$ & $1.256 \pm 0.075$ & $0.721 \pm 0.049$ & $0.358 \pm 0.021$ & $0.752 \pm 0.046$ & $0.977 \pm 0.059$ & $1.050 \pm 0.062$ \\
\hline $\log (\mathrm{Ne} / \mathrm{O})$ & $-0.784 \pm 0.033$ & $-0.830 \pm 0.039$ & $-0.907 \pm 0.034$ & $-0.809 \pm 0.036$ & $-0.763 \pm 0.033$ & $-0.797 \pm 0.032$ \\
\hline $\mathrm{S}^{+} / \mathrm{H}^{+} \times 10^{6}$ & $0.099 \pm 0.004$ & $\ldots$ & $0.026 \pm 0.001$ & $0.048 \pm 0.003$ & $0.094 \pm 0.003$ & $0.088 \pm 0.003$ \\
\hline $\mathrm{S}^{2+} / \mathrm{H}^{+} \times 10^{6}$ & $0.566 \pm 0.033$ & $\ldots$ & $0.152 \pm 0.011$ & $0.677 \pm 0.049$ & $0.462 \pm 0.025$ & $0.571 \pm 0.030$ \\
\hline $\mathrm{ICF}(\mathrm{S})$ & 1.777 & $\ldots$ & 2.639 & 1.908 & 1.543 & 1.737 \\
\hline $\mathrm{S} / \mathrm{H} \times 10^{6}$ & $1.181 \pm 0.059$ & $\cdots$ & $0.470 \pm 0.029$ & $1.385 \pm 0.094$ & $0.858 \pm 0.039$ & $1.144 \pm 0.053$ \\
\hline $\log (\mathrm{S} / \mathrm{O})$ & $-1.811 \pm 0.029$ & $\ldots$ & $-1.788 \pm 0.034$ & $-1.544 \pm 0.038$ & $-1.820 \pm 0.028$ & $-1.760 \pm 0.028$ \\
\hline $\mathrm{Cl}^{2+} / \mathrm{H}^{+} \times 10^{8}$ & $1.117 \pm 0.100$ & $\ldots$ & $\ldots$ & $\ldots$ & $0.584 \pm 0.050$ & $1.270 \pm 0.088$ \\
\hline $\mathrm{ICF}(\mathrm{Cl})$ & 1.504 & $\ldots$ & $\ldots$ & $\ldots$ & 1.489 & 1.510 \\
\hline $\mathrm{Cl} / \mathrm{H} \times 10^{8}$ & $1.680 \pm 0.150$ & $\ldots$ & $\ldots$ & $\ldots$ & $0.869 \pm 0.074$ & $1.918 \pm 0.133$ \\
\hline $\log (\mathrm{Cl} / \mathrm{O})$ & $-3.658 \pm 0.044$ & $\cdots$ & $\cdots$ & $\cdots$ & $-3.814 \pm 0.042$ & $-3.536 \pm 0.036$ \\
\hline $\mathrm{Ar}^{2+} / \mathrm{H}^{+} \times 10^{7}$ & $1.670 \pm 0.066$ & $0.727 \pm 0.049$ & $0.309 \pm 0.018$ & $1.203 \pm 0.075$ & $1.320 \pm 0.051$ & $1.543 \pm 0.060$ \\
\hline $\mathrm{Ar}^{3+} / \mathrm{H}^{+} \times 10^{7}$ & $1.475 \pm 0.081$ & $1.637 \pm 0.125$ & $0.683 \pm 0.040$ & $0.610 \pm 0.058$ & $1.016 \pm 0.050$ & $1.288 \pm 0.061$ \\
\hline $\mathrm{ICF}(\mathrm{Ar})$ & 1.372 & 2.223 & 1.771 & 1.630 & 1.329 & 1.401 \\
\hline $\mathrm{Ar} / \mathrm{H} \times 10^{7}$ & $2.291 \pm 0.144$ & $1.616 \pm 0.298$ & $0.546 \pm 0.078$ & $1.961 \pm 0.155$ & $1.753 \pm 0.095$ & $2.162 \pm 0.120$ \\
\hline $\log (\mathrm{Ar} / \mathrm{O})$ & $-2.523 \pm 0.034$ & $-2.479 \pm 0.084$ & $-2.723 \pm 0.066$ & $-2.393 \pm 0.042$ & $-2.509 \pm 0.031$ & $-2.484 \pm 0.031$ \\
\hline \multicolumn{7}{|l|}{ [Fe III] 4658: } \\
\hline $\mathrm{Fe}^{2+} / \mathrm{H}^{+} \times 10^{6}$ & $0.173 \pm 0.013$ & $\ldots$ & $0.102 \pm 0.010$ & $0.124 \pm 0.015$ & $0.124 \pm 0.008$ & $0.139 \pm 0.008$ \\
\hline $\mathrm{ICF}(\mathrm{Fe})$ & 14.187 & $\ldots$ & 24.636 & 20.767 & 12.555 & 14.789 \\
\hline $\mathrm{Fe} / \mathrm{H} \times 10^{6}$ & $2.451 \pm 0.189$ & $\ldots$ & $2.516 \pm 0.247$ & $2.580 \pm 0.307$ & $1.558 \pm 0.103$ & $2.057 \pm 0.115$ \\
\hline $\log (\mathrm{Fe} / \mathrm{O})$ & $-1.494 \pm 0.039$ & $\ldots$ & $-1.059 \pm 0.048$ & $-1.274 \pm 0.057$ & $-1.560 \pm 0.035$ & $-1.505 \pm 0.031$ \\
\hline \multicolumn{7}{|l|}{ [Fe III] 4986: } \\
\hline $\mathrm{Fe}^{2+} / \mathrm{H}^{+} \times 10^{6}$ & $\ldots$ & $\ldots$ & $\ldots$ & $\ldots$ & $0.119 \pm 0.008$ & $0.102 \pm 0.006$ \\
\hline $\mathrm{ICF}(\mathrm{Fe})$ & $\ldots$ & $\ldots$ & $\ldots$ & $\ldots$ & 12.555 & 14.789 \\
\hline $\mathrm{Fe} / \mathrm{H} \times 10^{6}$ & $\cdots$ & $\cdots$ & $\cdots$ & $\begin{array}{l}\cdots \\
\cdots\end{array}$ & $1.490 \pm 0.096$ & $1.505 \pm 0.087$ \\
\hline $\log (\mathrm{Fe} / \mathrm{O})$ & $\ldots$ & $\ldots$ & $\ldots$ & $\ldots$ & $-1.580 \pm 0.034$ & $-1.641 \pm 0.032$ \\
\hline
\end{tabular}

